\title{
Optimal controlled teleportation via several kinds of three-qubit states
}

\author{
GAO Ting ${ }^{1,2 \dagger}$, YAN FengLi ${ }^{2,3} \&$ LI YouCheng ${ }^{3}$ \\ ${ }^{1}$ College of Mathematics and Information Science, Hebei Normal University, Shijiazhuang 050016, China; \\ ${ }^{2}$ Max-Planck-Institut für Quantenoptik, Hans-Kopfermann-Str. 1, D-85748 Garching, Germany; \\ ${ }^{3}$ College of Physics and Information Engineering, Hebei Normal University, Shijiazhuang 050016, China
}

The probability of successful controlled teleportation of an unknown qubit using a general three-particle state is investigated. The analytic expressions of maximal probabilities via several kinds of tripartite states are given, including a tripartite Greenberger-Horne-Zeilinger state and a tripartite W-state.

controlled teleportation, three-qubit state, maximal probability

Bennett et al. ${ }^{[1]}$ showed that an arbitrary unknown state of a qubit could be teleported from a sender to a spatially distant receiver with the aid of long-range Einstein-Podolsky-Rosen (EPR) correlations and transmission of two bits of classical information. Since then, quantum teleportation has been developed by many researchers ${ }^{[2-18]}$ due to its important applications in quantum communication ${ }^{[19-21]}$ and quantum computation. In the past several years, quantum teleportation has also been experimentally demonstrated by several groups ${ }^{[22-24]}$.

The controlled quantum teleportation scheme was presented by Karlsson and Bourennane ${ }^{[12]}$. In the scheme, an unknown state was perfectly transported from one place to another via a previously shared Greenberger-Horne-Zeilinger (GHZ) state by means of local operations and classical communications (LOCC) under the permission of the third party. The signal state cannot be transmitted unless all three sides agree to cooperate. The controlled quantum teleportation is useful in networked quantum information processing and cryptographic conferencing ${ }^{[25-30]}$, as well as in controlled quantum secure direct communication ${ }^{[31]}$, and also has many other interesting applications, such as opening an account with a manager's permission in a network. Recently, much work on controlled quantum teleportation has also been done ${ }^{[13,14,17,18]}$, but it is only restricted to the special quantum channels, such as GHZ state or W state. If a nonmaximally entangled state is taken as a quantum channel, then one cannot teleport a qubit with unit probability and unit fidelity. However, it is possible to teleport a qubit with probability $p<1$, called prob-

Received February 18, 2008; accepted March 31, 2008 doi: $10.1007 / \mathrm{s} 11433-008-0165-8$

†Corresponding author (email: gaoting@hebtu.edu.cn)

Supported by the National Natural Science Foundation of China (Grant No. 10671054), the Key Project of Science and Technology Research of Ministry of Education of China (Grant No. 207011), and the Natural Science Foundation of Hebei Province, China (Grant No. 07M006) 
abilistic quantum teleportation ${ }^{[15,16]}$. More recently, the probabilistic scheme has been generalized to teleport $N$ qubits ${ }^{[10,11]}$.

The entanglement property lies at the very heart of quantum information theory ${ }^{[32]}$. The reason is that entanglement is a physical resource to perform some of the most important quantum information tasks, such as quantum teleportation, quantum computation, etc. In ref. [33], Verstraete, Popp, and Cirac introduced a new concept they called localizable entanglement (LE). This quantity not only has a very well defined physical meaning that treats entanglement as a true physical resource, but also establishes a very close connection between entanglement and correlation functions. The LE $E_{i j}$ is defined as the maximum of the average entanglement between the spins $i$ and $j$ over all possible outcomes:

$$
E_{i j}=\max _{\varepsilon} \sum_{s} p_{s} E\left(\left|\phi_{s}\right\rangle\right)
$$

where $p_{s}$ denotes the probability to obtain the two-spin state $\left|\phi_{s}\right\rangle$ after performing the measurement $|s\rangle$ in the rest of the system, and $E\left(\left|\phi_{s}\right\rangle\right)$ is the chosen measure of entanglement of $\left|\phi_{s}\right\rangle$. The determination of the LE is a formidable task because it involves optimization over all the possible local measurement strategies, and thus cannot be determined in general. However, Verstaete, Popp, and Cirac gave the tight upper bound and lower bound in the case of $E\left(\left|\phi_{s}\right\rangle\right)$ being the concurrence of $\left|\phi_{s}\right\rangle$. We determined the exact value of this kind of LE of the general tripartite state, and obtained the analytic expression of another kind of LE, i.e. the maximal success probability of controlled teleportation of a qubit of unknown information from a sender to a remote receiver via the control of a third agent using a general three-qubit state ${ }^{[34]}$.

In this paper, we give the exact values of the maximal probabilities of successful controlled teleportation of an unknown qubit via many kinds of tripartite states including a tripartite GHZ state and a tripartite $\mathrm{W}$-state.

\section{The controlled quantum teleportation using a general three-particle state}

Acín et al. ${ }^{[35]}$ gave the minimal decomposition of any pure three-qubit state in terms of orthogonal product states built from local bases - a generalization of the two-quantum-bit Schmidt decomposition. They proved that for any pure three-quantum-bit state there exist the local bases allowing one to build a set of five orthogonal product states in terms of which the state is written in a unique form. That is, for every pure state of a composite system, 1, 2 and 3, there exist orthonormal states $|0\rangle_{1},|1\rangle_{1}$ for system 1, orthonormal states $|0\rangle_{2},|1\rangle_{2}$ for system 2, and orthonormal states $|0\rangle_{3}$, $|1\rangle_{3}$ for system 3 such that

$$
\begin{gathered}
|\Psi\rangle_{123}=a_{0}|000\rangle_{123}+a_{1} \mathrm{e}^{\mathrm{i} \mu}|100\rangle_{123}+a_{2}|101\rangle_{123}+a_{3}|110\rangle_{123}+a_{4}|111\rangle_{123}, \\
a_{i} \geqslant 0, \quad 0 \leqslant \mu \leqslant \pi, \quad \sum_{i=0}^{4} a_{i}^{2}=1 .
\end{gathered}
$$

It is uniquely characterized by the five entanglement parameters.

Suppose that Alice is to deliver an unknown state to a distant receiver Bob supervised by the controller Charlie via a quantum channel of a normalized general pure three-qubit state in eq. (2), where particle 1 belongs to Charlie, particle 2 is in Alice's side, while Bob has particle 3. Let 
$a_{0} \neq 0$ throughout the paper, because if $a_{0}=0$, then $|\Psi\rangle_{123}$ is a tensor product state of a pure state of particle 1 and a pure state of particles 2 and 3, but not a true tripartite entangled state. Bob will get the qubit of quantum information carried by the unknown state only if he obtains the permission of Charlie (i.e., Charlie is trustworthy and cooperative).

After getting the approval of Charlie, Alice and Bob begin their teleportation under the control of Charlie.

The controller Charlie measures his particle in the basis

$$
|x\rangle=\cos \frac{\theta}{2}|0\rangle+\mathrm{e}^{\mathrm{i} \varphi} \sin \frac{\theta}{2}|1\rangle, \quad|x\rangle^{\perp}=\sin \frac{\theta}{2}|0\rangle-\mathrm{e}^{\mathrm{i} \varphi} \cos \frac{\theta}{2}|1\rangle,
$$

and broadcasts his measurement result. Here $\theta \in[0, \pi], \varphi \in[0,2 \pi]$.

The tripartite state $|\Psi\rangle_{123}$ is reexpressed as

$$
|\Psi\rangle_{123}=\sqrt{p_{1}}|x\rangle_{1}\left|\Phi_{1}\right\rangle_{23}+\sqrt{p_{2}}|x\rangle_{1}^{\perp}\left|\Phi_{2}\right\rangle_{23},
$$

where

$$
\begin{aligned}
p_{1}= & \sin ^{2} \frac{\theta}{2}+a_{0}^{2} \cos \theta+a_{0} a_{1} \cos (\mu-\varphi) \sin \theta, \\
p_{2}= & \cos ^{2} \frac{\theta}{2}-a_{0}^{2} \cos \theta-a_{0} a_{1} \cos (\mu-\varphi) \sin \theta, \\
\left|\Phi_{1}\right\rangle_{23}= & \frac{1}{\sqrt{p_{1}}}\left[\left(a_{0} \cos \frac{\theta}{2}+a_{1} \mathrm{e}^{\mathrm{i}(\mu-\varphi)} \sin \frac{\theta}{2}\right)|00\rangle_{23}\right. \\
& \left.+\mathrm{e}^{-\mathrm{i} \varphi} \sin \frac{\theta}{2}\left(a_{2}|01\rangle_{23}+a_{3}|10\rangle_{23}+a_{4}|11\rangle_{23}\right)\right], \\
\left|\Phi_{2}\right\rangle_{23}= & \frac{1}{\sqrt{p_{2}}}\left[\left(a_{0} \sin \frac{\theta}{2}-a_{1} \mathrm{e}^{\mathrm{i}(\mu-\varphi)} \cos \frac{\theta}{2}\right)|00\rangle_{23}\right. \\
& \left.-\mathrm{e}^{-\mathrm{i} \varphi} \cos \frac{\theta}{2}\left(a_{2}|01\rangle_{23}+a_{3}|10\rangle_{23}+a_{4}|11\rangle_{23}\right)\right] .
\end{aligned}
$$

After Charlie's measurement, the quantum channel is collapsed to $\left|\Phi_{1}\right\rangle_{23}$ and $\left|\Phi_{2}\right\rangle_{23}$ with probability $p_{1}$ and $p_{2}$.

By Schmidt decomposition,

$$
\begin{gathered}
\left|\Phi_{1}\right\rangle_{23}=\sqrt{\lambda_{10}}\left|0_{2}^{\prime} 0_{3}^{\prime}\right\rangle+\sqrt{\lambda_{11}}\left|1_{2}^{\prime} 1_{3}^{\prime}\right\rangle, \\
\left|\Phi_{2}\right\rangle_{23}=\sqrt{\lambda_{20}}\left|\overline{0}_{2} \overline{0}_{3}\right\rangle+\sqrt{\lambda_{21}}\left|\overline{1}_{2} \overline{1}_{3}\right\rangle,
\end{gathered}
$$

where $\left\{0_{2}^{\prime}, 1_{2}^{\prime}\right\}$ and $\left\{\overline{0}_{2}, \overline{1}_{2}\right\}\left(\left\{0_{3}^{\prime}, 1_{3}^{\prime}\right\}\right.$ and $\left.\left\{\overline{0}_{3}, \overline{1}_{3}\right\}\right)$ are orthonormal bases of system 2 (system 3), and Schmidt coefficients

$$
\lambda_{10}=\frac{1-\sqrt{1-C_{1}^{2}}}{2}, \quad \lambda_{11}=\frac{1+\sqrt{1-C_{1}^{2}}}{2}, \quad \lambda_{20}=\frac{1-\sqrt{1-C_{2}^{2}}}{2}, \quad \lambda_{21}=\frac{1+\sqrt{1-C_{2}^{2}}}{2},
$$

where

$$
C_{1}=\frac{\left|a_{0} a_{4} \mathrm{e}^{-\mathrm{i} \varphi} \sin \theta+2\left(a_{1} a_{4} \mathrm{e}^{\mathrm{i} \mu}-a_{2} a_{3}\right) \mathrm{e}^{-2 \mathrm{i} \varphi} \sin ^{2} \frac{\theta}{2}\right|}{p_{1}}
$$


and

$$
C_{2}=\frac{\left|a_{0} a_{4} \mathrm{e}^{-\mathrm{i} \varphi} \sin \theta-2\left(a_{1} a_{4} \mathrm{e}^{\mathrm{i} \mu}-a_{2} a_{3}\right) \mathrm{e}^{-2 \mathrm{i} \varphi} \cos ^{2} \frac{\theta}{2}\right|}{p_{2}}
$$

are the concurrence of $\left|\Phi_{1}\right\rangle_{23}$ and $\left|\Phi_{2}\right\rangle_{23}$.

For simplicity, we write eqs. (9) and (10) as

$$
\begin{aligned}
& \left|\Phi_{1}\right\rangle_{23}=\sqrt{\lambda_{10}}\left|0_{2} 0_{3}\right\rangle+\sqrt{\lambda_{11}}\left|1_{2} 1_{3}\right\rangle, \\
& \left|\Phi_{2}\right\rangle_{23}=\sqrt{\lambda_{20}}\left|0_{2} 0_{3}\right\rangle+\sqrt{\lambda_{21}}\left|1_{2} 1_{3}\right\rangle .
\end{aligned}
$$

Suppose that the unknown quantum state the sender Alice wants to teleport to Bob is

$$
|\psi\rangle_{4}=\alpha|0\rangle_{4}+\beta|1\rangle_{4}, \quad|\alpha|^{2}+|\beta|^{2}=1 .
$$

If the measurement outcome of Charlie is $|x\rangle_{1}$, then the collective state of particles 2, 3 and 4 is

$$
\begin{aligned}
|\psi\rangle_{4}\left|\Phi_{1}\right\rangle_{23}= & \sqrt{\lambda_{10}|\alpha|^{2}+\lambda_{11}|\beta|^{2}}\left[\frac { 1 } { \sqrt { 2 } } \left(\left|\phi^{+}\right\rangle_{24} \frac{\alpha \sqrt{\lambda_{10}}|0\rangle_{3}+\beta \sqrt{\lambda_{11}}|1\rangle_{3}}{\sqrt{\lambda_{10}|\alpha|^{2}+\lambda_{11}|\beta|^{2}}}\right.\right. \\
& \left.\left.+\left|\phi^{-}\right\rangle_{24} \frac{\alpha \sqrt{\lambda_{10}}|0\rangle_{3}-\beta \sqrt{\lambda_{11}}|1\rangle_{3}}{\sqrt{\lambda_{10}|\alpha|^{2}+\lambda_{11}|\beta|^{2}}}\right)\right] \\
& +\sqrt{\lambda_{11}|\alpha|^{2}+\lambda_{10}|\beta|^{2}}\left[\frac { 1 } { \sqrt { 2 } } \left(\left|\psi^{+}\right\rangle_{24} \frac{\beta \sqrt{\lambda_{10}}|0\rangle_{3}+\alpha \sqrt{\lambda_{11}}|1\rangle_{3}}{\sqrt{\lambda_{11}|\alpha|^{2}+\lambda_{10}|\beta|^{2}}}\right.\right. \\
& \left.\left.+\left|\psi^{-}\right\rangle_{24} \frac{\beta \sqrt{\lambda_{10}}|0\rangle_{3}-\alpha \sqrt{\lambda_{11}}|1\rangle_{3}}{\sqrt{\lambda_{11}|\alpha|^{2}+\lambda_{10}|\beta|^{2}}}\right)\right],
\end{aligned}
$$

where $\left|\phi^{ \pm}\right\rangle=\frac{1}{\sqrt{2}}(|00\rangle \pm|11\rangle), \quad\left|\psi^{ \pm}\right\rangle=\frac{1}{\sqrt{2}}(|01\rangle \pm|10\rangle)$. Alice makes a Bell measurement on her particles 2 and 4 . She obtains $\left|\phi^{+}\right\rangle_{24},\left|\phi^{-}\right\rangle_{24},\left|\psi^{+}\right\rangle_{24}$ and $\left|\psi^{-}\right\rangle_{24}$ with probability $\left(\frac{\sqrt{\lambda_{10}|\alpha|^{2}+\lambda_{11}|\beta|^{2}}}{\sqrt{2}}\right)^{2}, \quad\left(\frac{\sqrt{\lambda_{10}|\alpha|^{2}+\lambda_{11}|\beta|^{2}}}{\sqrt{2}}\right)^{2}, \quad\left(\frac{\sqrt{\lambda_{11}|\alpha|^{2}+\lambda_{10}|\beta|^{2}}}{\sqrt{2}}\right)^{2} \quad$ and $\left(\frac{\sqrt{\lambda_{11}|\alpha|^{2}+\lambda_{10}|\beta|^{2}}}{\sqrt{2}}\right)^{2}$. Then she conveys her measurement outcome to Bob over a classical communication channel.

In order to achieve teleportation, Bob needs to introduce an auxiliary particle $b$ with the initial 
state $|0\rangle_{b}$ and performs a collective unitary transformation $U_{3 b}=\left(\begin{array}{cccc}1 & 0 & 0 & 0 \\ 0 & 1 & 0 & 0 \\ 0 & 0 & \frac{\sqrt{\lambda_{10}}}{\sqrt{\lambda_{11}}} & \sqrt{1-\frac{\lambda_{10}}{\lambda_{11}}} \\ & & -\sqrt{1-\frac{\lambda_{10}}{\lambda_{11}}} & \frac{\sqrt{\lambda_{10}}}{\sqrt{\lambda_{11}}}\end{array}\right)$ on the state of particles 3 and $b$. Then the measurement on his auxiliary particle $b$ follows. If his measurement result is $|0\rangle_{b}$, Bob can fix up the state of his particle 3, recovering $|\psi\rangle$, by applying an appropriate local unitary operation. The achievable success probability of teleporting the unknown state in eq. (14) via $\left|\Phi_{1}\right\rangle_{23}$ is

$$
\begin{aligned}
& 2\left(\frac{\sqrt{\lambda_{10}|\alpha|^{2}+\lambda_{11}|\beta|^{2}}}{\sqrt{2}}\right)^{2}\left(\frac{\sqrt{\lambda_{10}}}{\sqrt{\lambda_{10}|\alpha|^{2}+\lambda_{11}|\beta|^{2}}}\right)^{2} \\
& +2\left(\frac{\sqrt{\lambda_{11}|\alpha|^{2}+\lambda_{10}|\beta|^{2}}}{\sqrt{2}}\right)^{2}\left(\frac{\sqrt{\lambda_{10}}}{\sqrt{\lambda_{11}|\alpha|^{2}+\lambda_{10}|\beta|^{2}}}\right)^{2}=2 \lambda_{10} .
\end{aligned}
$$

Similarly, if the measurement result of Charlie is $|x\rangle_{1}^{\perp}$, the achievable success probability of teleporting the state in eq. (14) via $\left|\Phi_{2}\right\rangle_{23}$ is $2 \lambda_{20}$.

Therefore, the probability $p$ of successful controlled teleportation of an unknown qubit (14) using a general three-particle state in eq. (2) is

$$
p=2 p_{1} \lambda_{10}+2 p_{2} \lambda_{20}=1-(\sqrt{P(\theta, \varphi)}+\sqrt{Q(\theta, \varphi)}),
$$

where

$$
\begin{aligned}
P(\theta, \varphi)= & p_{1}^{2}\left(1-C_{1}^{2}\right) \\
= & \frac{1}{4} a_{0}^{2} a_{1}^{2} \cos 2(\varphi-\mu)+3 a_{1} a_{2} a_{3} a_{4} \cos \mu \\
& +\frac{1}{8}\left(3-4 a_{0}^{2}+4 a_{0}^{4}+2 a_{0}^{2} a_{1}^{2}-12 a_{2}^{2} a_{3}^{2}-4 a_{0}^{2} a_{4}^{2}-12 a_{1}^{2} a_{4}^{2}\right) \\
& +\frac{1}{8} \cos 2 \theta\left[1-4 a_{0}^{2}+4 a_{0}^{4}-2 a_{0}^{2} a_{1}^{2}-2 a_{0}^{2} a_{1}^{2} \cos 2(\varphi-\mu)\right. \\
& \left.-4 a_{2}^{2} a_{3}^{2}+8 a_{1} a_{2} a_{3} a_{4} \cos \mu+4 a_{0}^{2} a_{4}^{2}-4 a_{1}^{2} a_{4}^{2}\right] \\
& -\cos \theta\left(\frac{1}{2}-a_{0}^{2}-2 a_{2}^{2} a_{3}^{2}+4 a_{1} a_{2} a_{3} a_{4} \cos \mu-2 a_{1}^{2} a_{4}^{2}\right) \\
& +a_{0} \sin \theta\left[2 a_{2} a_{3} a_{4} \cos \varphi+a_{1}\left(1-2 a_{4}^{2}\right) \cos (\varphi-\mu)\right] \\
& -\frac{1}{2} a_{0} \sin 2 \theta\left[2 a_{2} a_{3} a_{4} \cos \varphi+a_{1}\left(1-2 a_{0}^{2}-2 a_{4}^{2}\right) \cos (\varphi-\mu)\right], \\
Q(\theta, \varphi)= & p_{2}^{2}\left(1-C_{2}^{2}\right) \\
= & \frac{1}{4} a_{0}^{2} a_{1}^{2} \cos 2(\varphi-\mu)+3 a_{1} a_{2} a_{3} a_{4} \cos \mu
\end{aligned}
$$




$$
\begin{aligned}
& +\frac{1}{8}\left(3-4 a_{0}^{2}+4 a_{0}^{4}+2 a_{0}^{2} a_{1}^{2}-12 a_{2}^{2} a_{3}^{2}-4 a_{0}^{2} a_{4}^{2}-12 a_{1}^{2} a_{4}^{2}\right) \\
& +\frac{1}{8} \cos 2 \theta\left[1-4 a_{0}^{2}+4 a_{0}^{4}-2 a_{0}^{2} a_{1}^{2}-2 a_{0}^{2} a_{1}^{2} \cos 2(\varphi-\mu)\right. \\
& \left.-4 a_{2}^{2} a_{3}^{2}+8 a_{1} a_{2} a_{3} a_{4} \cos \mu+4 a_{0}^{2} a_{4}^{2}-4 a_{1}^{2} a_{4}^{2}\right] \\
& +\cos \theta\left(\frac{1}{2}-a_{0}^{2}-2 a_{2}^{2} a_{3}^{2}+4 a_{1} a_{2} a_{3} a_{4} \cos \mu-2 a_{1}^{2} a_{4}^{2}\right) \\
& -a_{0} \sin \theta\left[2 a_{2} a_{3} a_{4} \cos \varphi+a_{1}\left(1-2 a_{4}^{2}\right) \cos (\varphi-\mu)\right] \\
& -\frac{1}{2} a_{0} \sin 2 \theta\left[2 a_{2} a_{3} a_{4} \cos \varphi+a_{1}\left(1-2 a_{0}^{2}-2 a_{4}^{2}\right) \cos (\varphi-\mu)\right] .
\end{aligned}
$$

Thus

$$
\begin{array}{lll}
Q(\theta, \varphi)=P(\pi-\theta, \varphi+\pi), & \text { if } \quad \varphi \in[0, \pi], \\
Q(\theta, \varphi)=P(\pi-\theta, \varphi-\pi), & \text { if } \quad \varphi \in[\pi, 2 \pi], \\
P(\theta, \varphi)=Q(\pi-\theta, \varphi+\pi), & \text { if } \quad \varphi \in[0, \pi], \\
P(\theta, \varphi)=Q(\pi-\theta, \varphi-\pi), & \text { if } \quad \varphi \in[\pi, 2 \pi],
\end{array}
$$

and

$$
\begin{aligned}
& \sqrt{P(0, \varphi)}+\sqrt{Q(0, \varphi)}=\sqrt{P(\pi, \varphi)}+\sqrt{Q(\pi, \varphi)}, \\
& \sqrt{P(\theta, 0)}+\sqrt{Q(\theta, 0)}=\sqrt{P(\theta, 2 \pi)}+\sqrt{Q(\theta, 2 \pi)}
\end{aligned}
$$

It is clear that the success probability is the same as the above eq. (16) if the operation order is changed. That is, if Alice makes a Bell state measurement on her particles 2 and 4 first, the third party Charlie's measurement on his particle 1 follows. After that Bob operates his particle to acquire a qubit of quantum information Alice sends, instead of the above operation order. And then they also achieve the success probability (16) of controlled teleportation.

\section{The maximal success probability of controlled quantum teleportation using three-particle states with $a_{1} a_{2} a_{3} a_{4} \sin \mu=0$}

In ref. [34], we determined the analytic expression of the maximal success probability of controlled teleportation by using the general tripartite state (2) with $a_{0} a_{1} a_{2} a_{3} a_{4} \sin \mu \neq 0$. In this section, we give the analytic expression of the localizable entanglement (LE), i.e. the maximal probability of successful controlled teleportation of an unknown qubit state (14) via every three-qubit state (2) satisfying $a_{1} a_{2} a_{3} a_{4} \sin \mu=0$, and investigate how to achieve it (that is, Charlie finds an optimal measurement basis).

It is clear that the maximum of eq. (16) is

$$
p_{\max }=\max \{p\}=\max \left\{2 p_{1} \lambda_{10}+2 p_{2} \lambda_{20}\right\}=1-\min \{\sqrt{P(\theta, \varphi)}+\sqrt{Q(\theta, \varphi)}\} .
$$

In order to get the maximum $p_{\max }$ of $p$ in eq. (22), we need only obtain the minimum of $\sqrt{P(\theta, \varphi)}+\sqrt{Q(\theta, \varphi)}$ :

$$
\min \{\sqrt{P(\theta, \varphi)}+\sqrt{Q(\theta, \varphi)}\} .
$$

In other words, to reach the maximal probability of controlled teleportation through an arbitrary 
partially entangled quantum channel (2), the supervisor Charlie only needs to choose an optimal measurement basis, i.e. he selects $\theta_{0}$ and $\varphi_{0}$ such that $\min \{\sqrt{P(\theta, \varphi)}+\sqrt{Q(\theta, \varphi)}\}=$ $\sqrt{P\left(\theta_{0}, \varphi_{0}\right)}+\sqrt{Q\left(\theta_{0}, \varphi_{0}\right)}$

Note that the minimum of $\sqrt{P(\theta, \varphi)}+\sqrt{Q(\theta, \varphi)}$ should occur at the points such that $P(\theta, \varphi)=0, Q(\theta, \varphi)=0$,

$$
\begin{aligned}
& \frac{\partial(\sqrt{P(\theta, \varphi)}+\sqrt{Q(\theta, \varphi)})}{\partial \theta}=0, \\
& \frac{\partial(\sqrt{P(\theta, \varphi)}+\sqrt{Q(\theta, \varphi)})}{\partial \varphi}=0,
\end{aligned}
$$

or at the boundary of $\theta$ and $\varphi$. From eqs. (24) and (25), there are

$$
\begin{gathered}
\frac{\partial P(\theta, \varphi)}{\partial \theta} \frac{\partial Q(\theta, \varphi)}{\partial \varphi}-\frac{\partial Q(\theta, \varphi)}{\partial \theta} \frac{\partial P(\theta, \varphi)}{\partial \varphi}=0, \\
P(\theta, \varphi)\left(\frac{\partial Q(\theta, \varphi)}{\partial \varphi}\right)^{2}-Q(\theta, \varphi)\left(\frac{\partial P(\theta, \varphi)}{\partial \varphi}\right)^{2}=0, \\
P(\theta, \varphi)\left(\frac{\partial Q(\theta, \varphi)}{\partial \theta}\right)^{2}-Q(\theta, \varphi)\left(\frac{\partial P(\theta, \varphi)}{\partial \theta}\right)^{2}=0 .
\end{gathered}
$$

Let $y=\cot \theta, t=\cot \frac{\theta}{2}, \theta \in(0, \pi)$, then $\sin \theta=\frac{1}{\sqrt{y^{2}+1}}=\frac{2 t}{1+t^{2}}, \quad \cos \theta=\frac{y}{\sqrt{y^{2}+1}}=\frac{t^{2}-1}{t^{2}+1}$, $t=y+\sqrt{y^{2}+1}, \quad t \in(0,+\infty)$. These will be useful throughout the paper. Next we give the controller's optimal measurement basis (3) for every kind of given quantum channel (2) satisfying $a_{1} a_{2} a_{3} a_{4} \sin \mu=0$. That is, we determine the two parameters $\theta$ and $\varphi$ in measurement basis (3). To state clearly, we classify the quantum channel (2) into the following cases. Next we examine quantum channels (2) with the following different characterizations, give the maximal probability of teleportation via any three-qubit state (2) with $a_{1} a_{2} a_{3} a_{4} \sin \mu=0$, and characterize the tripartite states that can collapse to an EPR pair after Charlie's measurement.

\section{$2.1 \quad a_{1}=a_{2}=a_{3}=0$ and $a_{0} a_{4 \neq 0}$}

The quantum channel (2) with three coefficients being 0 is the only one satisfying $a_{1}=a_{2}=a_{3}=0$ and $a_{0} a_{4} \neq 0$, because others are biseparable (one party is not entangled with the other two parties) and cannot be used as a quantum channel of controlled teleportation.

It is seen that

$$
\begin{aligned}
\min \{\sqrt{P(\theta, \varphi)}+\sqrt{Q(\theta, \varphi)}\} & =\min \left\{\sqrt{\frac{1}{4}\left[\cos \theta-\left(1-2 a_{0}^{2}\right)\right]^{2}}+\sqrt{\frac{1}{4}\left[\cos \theta+\left(1-2 a_{0}^{2}\right)\right]^{2}}\right\} \\
& =\left|1-2 a_{0}^{2}\right|
\end{aligned}
$$

for each $\varphi \in[0,2 \pi]$ and all $\theta$ satisfying $|\cos \theta| \leqslant\left|1-2 a_{0}^{2}\right|$, which implies that 


$$
p_{\max }=1-\left|1-2 a_{0}^{2}\right| .
$$

Note that if $\cos \theta=1-2 a_{0}^{2} \neq 0$ or $\cos \theta=-1+2 a_{0}^{2} \neq 0$, then $P(\theta, \varphi)=0$ or $Q(\theta, \varphi)=0$, which means that after Charlie's measurement the state of particles 2 and 3 is an EPR pair with probability $2 a_{0}^{2}\left(1-a_{0}^{2}\right)$. Moreover, if $\cos \theta=1-2 a_{0}^{2}=0$ or $\cos \theta=-1+2 a_{0}^{2}=0$, i.e. $\theta=\frac{\pi}{2}$, $a_{0}=a_{4}=\frac{1}{\sqrt{2}}$, then $P(\theta, \varphi)=Q(\theta, \varphi)=0$, that is, after Charlie measures the quantum channel in the basis $\left\{\frac{|0\rangle+\mathrm{e}^{\mathrm{i} \varphi}|1\rangle}{\sqrt{2}}, \frac{|0\rangle-\mathrm{e}^{\mathrm{i} \varphi}|1\rangle}{\sqrt{2}}\right\}$, particles 2 and 3 are collapsed to a Bell state with probability 1 . It follows that perfect quantum teleportation can be achieved if $a_{0}=\frac{1}{\sqrt{2}}$ (i.e. the quantum channel is in GHZ state) and $\theta=\frac{\pi}{2}$. That is, one can send a perfect unknown state to another using GHZ state as a quantum channel via the controller's measurement in the basis $\left\{\frac{|0\rangle+\mathrm{e}^{\mathrm{i} \varphi}|1\rangle}{\sqrt{2}}, \frac{|0\rangle-\mathrm{e}^{\mathrm{i} \varphi}|1\rangle}{\sqrt{2}}\right\}$, where $\varphi \in[0,2 \pi]$.

\section{$2.2 a_{1}=a_{4}=0$ and $a_{0} a_{2} a_{3} \neq 0$}

Here we consider the quantum channel with coefficients satisfying $a_{1}=a_{4}=0$ and $a_{0} a_{2} a_{3} \neq 0$. These states are called tri-Bell states in ref. [35]. Note that W-state

$$
|W\rangle=\frac{1}{\sqrt{3}}(|001\rangle+|010\rangle+|100\rangle)
$$

is contained here, because the quantum channel (2) in the case of $a_{1}=a_{4}=0$ and $a_{0}=a_{2}=a_{3}=\frac{1}{\sqrt{3}}$ is LOCC equivalent to W-state.

If $P(\theta, \varphi)=0$, then $\cos \theta=\frac{1+2 a_{2} a_{3}}{1-2 a_{0}^{2}+2 a_{2} a_{3}}$, or $\cos \theta=\frac{-1+2 a_{2} a_{3}}{-1+2 a_{0}^{2}+2 a_{2} a_{3}}$. It is not difficult to prove that $\quad\left|\frac{1+2 a_{2} a_{3}}{1-2 a_{0}^{2}+2 a_{2} a_{3}}\right|>1, \quad\left|\frac{-1+2 a_{2} a_{3}}{-1+2 a_{0}^{2}+2 a_{2} a_{3}}\right|>1 \quad$ if $\quad a_{2} \neq a_{3}$, and $\frac{-1+2 a_{2} a_{3}}{-1-2 a_{0}^{2}+2 a_{2} a_{3}}=-1$ if $a_{2}=a_{3}$. Thus, $P(\theta, \varphi)=0$ if and only if $a_{2}=a_{3}$ and $\theta=\pi$. From eq. (19), there is $Q(\theta, \varphi)=0$ if and only if $a_{2}=a_{3}$ and $\theta=0$. Combining eqs. (19) and (20), we have

$$
\left.(\sqrt{P(\theta, \varphi)}+\sqrt{Q(\theta, \varphi)})\right|_{P(\theta, \varphi)=0}=\left.(\sqrt{P(\theta, \varphi)}+\sqrt{Q(\theta, \varphi)})\right|_{Q(\theta, \varphi)=0}=a_{0}^{2} .
$$

Therefore, the quantum channel (2) satisfying $a_{1}=a_{4}=0, a_{2}=a_{3}$ and $a_{0} a_{2} a_{3} \neq 0$ can be collapsed to a Bell state with probability $p_{1}=p_{2}=1-a_{0}^{2}$ by Charlie measuring his particle in the basis $\{|0\rangle,|1\rangle\}$. 
Next we suppose that $P(\theta, \varphi) \neq 0$ and $Q(\theta, \varphi) \neq 0$. From eq. (28), we derive that $a_{0}^{4} a_{2}^{2} a_{3}^{2}\left(-1+2 a_{0}^{2}+4 a_{2}^{2} a_{3}^{2}\right) \cos \theta \sin ^{2} \theta=0$. Thus, $\theta=0, \frac{\pi}{2}, \pi$. By checking, $\theta=0, \frac{\pi}{2}, \pi$ are roots of eq. (24). Note that if $\theta=0, \pi$, then $a_{2} \neq a_{3}$ by $P(\theta, \varphi) \neq 0$ and $Q(\theta, \varphi) \neq 0$. It is seen that $\sqrt{P(\theta, \varphi)}+\sqrt{Q(\theta, \varphi)}=a_{0}^{2}+\left|a_{2}^{2}-a_{3}^{2}\right|>0 \quad$ if $\quad \theta=0, \pi \quad$ and $\quad a_{2} \neq a_{3} ; \quad \sqrt{P(\theta, \varphi)}+\sqrt{Q(\theta, \varphi)}=$ $\sqrt{1-4 a_{2}^{2} a_{3}^{2}}>0$ if $\theta=\frac{\pi}{2}$. Therefore,

$$
\min \{\sqrt{P(\theta, \varphi)}+\sqrt{Q(\theta, \varphi)}\}=\min \left\{a_{0}^{2}+\left|a_{2}^{2}-a_{3}^{2}\right|, \sqrt{1-4 a_{2}^{2} a_{3}^{2}}\right\}>0 .
$$

That is, for the quantum channel (2) with $a_{1}=a_{4}=0, a_{0} a_{2} a_{3} \neq 0$ and $a_{0}^{2}+\left|a_{2}^{2}-a_{3}^{2}\right|<$ $\sqrt{1-4 a_{2}^{2} a_{3}^{2}}$, the controller should choose the measurement basis $\{|0\rangle,|1\rangle\}$; otherwise, he selects the measurement basis (3) with $\theta=\frac{\pi}{2}$ (i.e. he measures in the basis $\left\{\frac{|0\rangle+\mathrm{e}^{\mathrm{i} \varphi}|1\rangle}{\sqrt{2}}\right.$, $\left.\frac{|0\rangle-\mathrm{e}^{\mathrm{i} \varphi}|1\rangle}{\sqrt{2}}\right\}$, where $\varphi \in[0,2 \pi]$, thus the controlled teleportation in sec. 1 achieves the maximal success probability $p_{\max }<1$.

\subsection{One is $a_{1}=a_{2}=0$ and $a_{0} a_{3} a_{4} \neq 0$; the other is $a_{1}=a_{3}=0$ and $a_{0} a_{2} a_{4} \neq 0$}

Now we discuss the quantum channel (2) with the characterization $a_{1}=a_{2}=0$ and $a_{0} a_{3} a_{4} \neq 0$.

First, $P(\theta, \varphi) \neq 0$ and $Q(\theta, \varphi) \neq 0$. If $P(\theta, \varphi)=0$, then $\cos \theta=\frac{1-2 a_{0}^{2}-4 a_{0}^{2} a_{4} \sqrt{-a_{3}^{2}}}{1-4 a_{0}^{2}+4 a_{0}^{4}+4 a_{0}^{2} a_{4}^{2}}$, or $\cos \theta=\frac{1-2 a_{0}^{2}+4 a_{0}^{2} a_{4} \sqrt{-a_{3}^{2}}}{1-4 a_{0}^{2}+4 a_{0}^{4}+4 a_{0}^{2} a_{4}^{2}}$, which are impossible. Thus, $P(\theta, \varphi) \neq 0$. By eq. (19), there is also $Q(\theta, \varphi) \neq 0$. The quantum channel (2) with the characterization $a_{1}=a_{2}=0$ and $a_{0} a_{3} a_{4} \neq 0$ can never collapse to an EPR pair after Charlie's measurement. Second, eq. (28) implies $a_{0}^{4}\left(1-2 a_{0}^{2}\right) a_{4}^{2} a_{3}^{2} \cos \theta \sin ^{2} \theta=0$. It follows that $\theta=0, \frac{\pi}{2}, \pi$. By checking, $\theta=0, \frac{\pi}{2}, \pi$ are the roots of eq. (24). We get

$$
\begin{aligned}
\min \{\sqrt{P(\theta, \varphi)}+\sqrt{Q(\theta, \varphi)}\} & =\sqrt{P\left(\frac{\pi}{2}, \varphi\right)}+\sqrt{Q\left(\frac{\pi}{2}, \varphi\right)}=\sqrt{1-4 a_{0}^{2} a_{4}^{2}}>0, \\
p_{\max } & =1-\sqrt{1-4 a_{0}^{2} a_{4}^{2}}<1,
\end{aligned}
$$

where $\theta=\frac{\pi}{2}$. Similarly, when $a_{1}=a_{3}=0$, and $a_{0} a_{2} a_{4} \neq 0$, we have

$$
p_{\max }=1-\sqrt{1-4 a_{0}^{2} a_{4}^{2}}<1
$$

where $\theta=\frac{\pi}{2}$. That is, the controlled teleportation via the quantum channel (2) satisfying 
$a_{1}=a_{2}=0$ and $a_{0} a_{3} a_{4} \neq 0$, or $a_{1}=a_{3}=0$ and $a_{0} a_{2} a_{4} \neq 0$, can only succeed with optimal probability $p_{\max }=1-\sqrt{1-4 a_{0}^{2} a_{4}^{2}}<1$.

\subsection{One is $a_{2}=a_{4}=0$ and $a_{0} a_{1} a_{3} \neq 0$; the other is $a_{3}=a_{4}=0$ and $a_{0} a_{1} a_{2} \neq 0$}

For the quantum channel with characterization either $a_{2}=a_{4}=0$ and $a_{0} a_{1} a_{3} \neq 0$, or $a_{3}=a_{4}$ $=0$ and $a_{0} a_{1} a_{2} \neq 0$, because $P(\theta, \varphi)=p_{1}^{2}, Q(\theta, \varphi)=p_{2}^{2}$, there are $\min \{\sqrt{P(\theta, \varphi)}+\sqrt{Q(\theta, \varphi)}\}$ $=1$ and $p_{\max }=0$ for both cases. This is also seen directly from the quantum channel (2) with these two characterizations being biseparable states.

\section{$2.5 \quad a_{2}=a_{3}=0$ and $a_{0} a_{1} a_{4} \neq 0$}

The quantum channel (2) with coefficients satisfying $a_{2}=a_{3}=0$, but $a_{0} a_{1} a_{4} \neq 0$, is extended GHZ states according to the classification in ref. [35].

For this kind of quantum channel, we derive that

$$
\begin{gathered}
P(\theta, \varphi)=\frac{1}{4}\left[1-2 a_{4}^{2}+2 a_{0} a_{1} \cos (\varphi-\mu) \sin \theta-\left(1-2 a_{0}^{2}-2 a_{4}^{2}\right) \cos \theta\right]^{2}, \\
Q(\theta, \varphi)=\frac{1}{4}\left[-1+2 a_{4}^{2}+2 a_{0} a_{1} \cos (\varphi-\mu) \sin \theta-\left(1-2 a_{0}^{2}-2 a_{4}^{2}\right) \cos \theta\right]^{2},
\end{gathered}
$$

and

$$
p_{\max }=1-\min \{\sqrt{P(\theta, \varphi)}+\sqrt{Q(\theta, \varphi)}\}=1-\left|1-2 a_{4}^{2}\right|,
$$

where $\theta, \varphi$ satisfy $\left|2 a_{0} a_{1} \cos (\varphi-\mu) \sin \theta-\left(1-2 a_{0}^{2}-2 a_{4}^{2}\right) \cos \theta\right| \leqslant\left|1-2 a_{4}^{2}\right|$. Note that the set $S$ of $(\theta, \varphi)$ satisfying

$$
\left|2 a_{0} a_{1} \cos (\varphi-\mu) \sin \theta-\left(1-2 a_{0}^{2}-2 a_{4}^{2}\right) \cos \theta\right| \leqslant\left|1-2 a_{4}^{2}\right|
$$

is a region, and we see that $\left(\frac{\pi}{2}, \frac{\pi}{2}+\mu\right),\left(\frac{\pi}{2}, \frac{3 \pi}{2}+\mu\right) \in S$ in the case of $0 \leqslant \mu \leqslant \frac{\pi}{2}$, and $\left(\frac{\pi}{2}, \frac{\pi}{2}+\mu\right),\left(\frac{\pi}{2},-\frac{\pi}{2}+\mu\right)$ in the case of $\frac{\pi}{2} \leqslant \mu \leqslant \pi$.

From eqs. (37) and (38), we have $P(\theta, \varphi)=Q(\theta, \varphi)=0$ if and only if $a_{4}=\frac{1}{\sqrt{2}}$ and $a_{1} \cos (\varphi-\mu) \sin \theta+a_{0} \cos \theta=0$, which means that as long as Charlie measures the quantum channel

$$
a_{0}|000\rangle+a_{1} \mathrm{e}^{\mathrm{i} \mu}|100\rangle+a_{4}|111\rangle, \quad a_{0}^{2}+a_{1}^{2}=a_{4}^{2}=\frac{1}{2},
$$

in the basis (3) satisfying $a_{1} \cos (\varphi-\mu) \sin \theta+a_{0} \cos \theta=0$, where $\theta \in[0, \pi], \varphi \in[0,2 \pi]$, Alice and Bob can obtain an EPR pair with certainty (i.e. with probability $p=1$ ), and Alice teleports her one-qubit information to Bob with probability 1 and unit fidelity. Note that the state

$$
a_{0}|000\rangle+a_{1}|100\rangle+a_{4}|111\rangle, \quad a_{0}^{2}+a_{1}^{2}=a_{4}^{2}=\frac{1}{2},
$$

is LOCC equivalent to the state in eq. (40). That is, the states in eq. (40) or (41) can be used for 
perfect controlled quantum teleportation. Thus, one achieves perfect teleportation via these states by the controller's measurement on his particle using the basis (3) satisfying $a_{1} \cos (\varphi-\mu) \sin \theta+a_{0} \cos \theta=0$. More important, this kind of states in eq. (41) or (40) is different from GHZ state according to the classification in ref. [35].

\section{$2.6 a_{1}=0$ and $a_{0} a_{2} a_{3} a_{4} \neq 0$}

In this section, we investigate the quantum channel with coefficients satisfying $a_{1}=0$ and $a_{0} a_{2} a_{3} a_{4} \neq 0$. Evidently,

$$
\begin{aligned}
\sqrt{P(0, \varphi)}+\sqrt{Q(0, \varphi)} & =\sqrt{P(\pi, \varphi)}+\sqrt{Q(\pi, \varphi)} \\
& =a_{0}^{2}+\sqrt{\left(1-a_{0}^{2}\right)^{2}-4 a_{2}^{2} a_{3}^{2}} \\
& =a_{0}^{2}+\sqrt{\left[a_{4}^{2}+\left(a_{2}-a_{3}\right)^{2}\right]\left[a_{4}^{2}+\left(a_{2}+a_{3}\right)^{2}\right]},
\end{aligned}
$$

and $P(\theta, \varphi) Q(\theta, \varphi) \neq 0$ in the case of $\theta=0, \pi$.

We first examine the condition of $P(\theta, \varphi)=0$. If $P(\theta, \varphi)=0$, then

$$
\cos \varphi=\frac{-\frac{1}{4}+a_{2}^{2} a_{3}^{2}+a_{0}^{2} a_{4}^{2}+\cos \theta\left(\frac{1}{2}-a_{0}^{2}-2 a_{2}^{2} a_{3}^{2}\right)-\frac{1}{4} \cos ^{2} \theta\left(1-4 a_{0}^{2}+4 a_{0}^{4}-4 a_{2}^{2} a_{3}^{2}+4 a_{0}^{2} a_{4}^{2}\right)}{2 \sin \theta(1-\cos \theta) a_{0} a_{2} a_{3} a_{4}} .
$$

Note that

$$
\begin{aligned}
z & =\frac{-\frac{1}{4}+a_{2}^{2} a_{3}^{2}+a_{0}^{2} a_{4}^{2}+\cos \theta\left(\frac{1}{2}-a_{0}^{2}-2 a_{2}^{2} a_{3}^{2}\right)-\frac{1}{4} \cos ^{2} \theta\left(1-4 a_{0}^{2}+4 a_{0}^{4}-4 a_{2}^{2} a_{3}^{2}+4 a_{0}^{2} a_{4}^{2}\right)}{2 \sin \theta(1-\cos \theta) a_{0} a_{2} a_{3} a_{4}} \\
& =\frac{-a_{0}^{4} t^{4}+\left(-2 a_{0}^{2}+2 a_{0}^{4}+4 a_{0}^{2} a_{4}^{2}\right) t^{2}-\left(1-a_{0}^{2}\right)^{2}+4 a_{2}^{2} a_{3}^{2}}{8 t a_{0} a_{2} a_{3} a_{4}}
\end{aligned}
$$

tends to $-\infty$ when $t$ tends to 0 and $+\infty$, and $z=-1$ holds only if $a_{2}=a_{3}$ and $t=\frac{a_{4}}{a_{0}}$. It follows that $z \leqslant-1$, where the equality occurs if and only if $a_{2}=a_{3}$ and $\theta=\theta_{0}$, where $\cot \frac{\theta_{0}}{2}=\frac{a_{4}}{a_{0}}$ and $0<\theta_{0}<\pi$. Therefore, only if $a_{2}=a_{3}, \varphi=\pi, \theta=\theta_{0}$, there is $P(\theta, \varphi)=0$.

By eqs. (19) and (20), we have $Q(\theta, \varphi)=0$ iff $a_{2}=a_{3}, \varphi=0$, and $\theta=\pi-\theta_{0}$, and

$$
\begin{aligned}
(\sqrt{P(\theta, \varphi)}+\sqrt{Q(\theta, \varphi)}) & \left.\right|_{P(\theta, \varphi)=0}=\left.\sqrt{Q\left(\theta_{0}, \pi\right)}\right|_{a_{2}=a_{3}} \\
& =\left.(\sqrt{P(\theta, \varphi)}+\sqrt{Q(\theta, \varphi)})\right|_{Q(\theta, \varphi)=0} \\
& =\left.\sqrt{P\left(\pi-\theta_{0}, 0\right)}\right|_{a_{2}=a_{3}} \\
& =\frac{\sqrt{a_{0}^{8}+2 a_{0}^{4} a_{4}^{2}-2 a_{0}^{6} a_{4}^{2}+a_{4}^{4}-2 a_{0}^{2} a_{4}^{4}-3 a_{0}^{4} a_{4}^{4}+8 a_{0}^{2} a_{3}^{2} a_{4}^{4}-4 a_{3}^{4} a_{4}^{4}}}{a_{0}^{2}+a_{4}^{2}} \\
& =\sqrt{1-4 a_{3}^{2}+4 a_{3}^{4}-4 a_{4}^{2}+12 a_{3}^{2} a_{4}^{2}+4 a_{4}^{4}} .
\end{aligned}
$$


That is, $\quad \sqrt{P(\theta, \varphi)}+\sqrt{Q(\theta, \varphi)}=\sqrt{1-4 a_{3}^{2}+4 a_{3}^{4}-4 a_{4}^{2}+12 a_{3}^{2} a_{4}^{2}+4 a_{4}^{4}} \quad$ if $\quad P(\theta, \varphi)=0 \quad$ or $Q(\theta, \varphi)=0$.

It is shown that an EPR pair is obtained with probability $p_{1}=p_{2}=\frac{a_{0}^{2}\left(1-a_{0}^{2}+a_{4}^{2}\right)}{a_{0}^{2}+a_{4}^{2}}$ if $a_{2}=$ $a_{3}$ and Charlie uses the measurement basis (3) with $(\theta, \varphi)=\left(\theta_{0}, \pi\right)$, or $(\theta, \varphi)=\left(\pi-\theta_{0}, 0\right)$, where $\cot \frac{\theta_{0}}{2}=\frac{a_{4}}{a_{0}}$ and $0<\theta_{0}<\pi$.

Next we assume that $P(\theta, \varphi) Q(\theta, \varphi) \neq 0$. From eqs. (26) and (27), there are

$$
\sin \varphi\left[2 a_{2} a_{3} a_{4} \cos \varphi \sin \theta-2 a_{0}^{3} \cos \theta+a_{0} \cos \theta\left(1-2 a_{4}^{2}\right)\right]=0
$$

and

$$
\sin \varphi\left[a_{0}\left(1-2 a_{4}^{2}\right) \cos \theta \sin ^{2} \theta+2 a_{0}^{3} \cos ^{3} \theta+2 a_{2} a_{3} a_{4} \cos \varphi \sin ^{3} \theta\right]=0 .
$$

By

$$
2 a_{2} a_{3} a_{4} \cos \varphi \sin \theta-2 a_{0}^{3} \cos \theta+a_{0} \cos \theta\left(1-2 a_{4}^{2}\right)=0,
$$

and

$$
a_{0}\left(1-2 a_{4}^{2}\right) \cos \theta \sin ^{2} \theta+2 a_{0}^{3} \cos ^{3} \theta+2 a_{2} a_{3} a_{4} \cos \varphi \sin ^{3} \theta=0,
$$

there are $\theta=\frac{\pi}{2}, \varphi=\frac{\pi}{2}, \frac{3 \pi}{2}$. Then

$$
\sqrt{P\left(\frac{\pi}{2}, \frac{\pi}{2}\right)}+\sqrt{Q\left(\frac{\pi}{2}, \frac{\pi}{2}\right)}=\sqrt{P\left(\frac{\pi}{2}, \frac{3 \pi}{2}\right)}+\sqrt{Q\left(\frac{\pi}{2}, \frac{3 \pi}{2}\right)}=\sqrt{1-4 a_{2}^{2} a_{3}^{2}-4 a_{0}^{2} a_{4}^{2}} .
$$

Next we only need to examine the case $\sin \varphi=0$, i.e. $\varphi=0, \pi, 2 \pi$, while $P(\theta, \varphi) Q(\theta, \varphi) \neq 0$ and $\theta \in(0, \pi)$.

Now we consider the case $\varphi=0$. From eq. (28), we obtain

$$
\begin{aligned}
& -4 a_{0}^{3}\left(2 y a_{0} a_{2} a_{3}+a_{4}\right)\left[4 y^{2} a_{0}^{2} a_{2} a_{3} a_{4}^{2}+2 y a_{0}\left(2 a_{0}^{4}+2 a_{0}^{2} a_{4}^{2}-3 a_{0}^{2}-4 a_{2}^{2} a_{3}^{2}-a_{4}^{2}+1\right) a_{4}\right. \\
& \left.\quad+a_{2} a_{3}\left(2 a_{0}^{2}-4 a_{0}^{2} a_{4}^{2}+4 a_{2}^{2} a_{3}^{2}+2 a_{4}^{2}-1\right)\right]=0,
\end{aligned}
$$

which implies that

$$
\begin{aligned}
& y=\cot \theta_{1}=-\frac{a_{4}}{2 a_{0} a_{2} a_{3},} \\
& y=\cot \theta_{2}=\left\{\begin{array}{lll}
\frac{a_{3}\left(1-2 a_{3}^{2}-2 a_{4}^{2}\right)}{2 a_{0} a_{2} a_{4}}, & \text { if } & a_{2}>a_{3}, \\
\frac{a_{2}\left(1-2 a_{2}^{2}-2 a_{4}^{2}\right)}{2 a_{0} a_{3} a_{4}}, & \text { if } & a_{2}<a_{3},
\end{array}\right. \\
& y=\cot \theta_{3}=\left\{\begin{array}{lll}
\frac{a_{2}\left(1-2 a_{2}^{2}-2 a_{4}^{2}\right)}{2 a_{0} a_{3} a_{4}}, & \text { if } & a_{2}>a_{3}, \\
\frac{a_{3}\left(1-2 a_{3}^{2}-2 a_{4}^{2}\right)}{2 a_{0} a_{2} a_{4}}, & \text { if } & a_{2}<a_{3} .
\end{array}\right.
\end{aligned}
$$

Note that when $a_{2}=a_{3}$, there is 


$$
\cot \theta_{2}=\cot \theta_{3}=\cot \left(\pi-\theta_{0}\right)=\frac{1-2 a_{3}^{2}-2 a_{4}^{2}}{2 a_{0} a_{4}}=\frac{a_{0}^{2}-a_{4}^{2}}{2 a_{0} a_{4}},
$$

which implies that $\theta_{2}=\theta_{3}=\pi-\theta_{0}$ (i.e. $\left.Q\left(\theta_{2}, 0\right)=0\right)$ for $\theta_{2}, \theta_{3}, \pi-\theta_{0} \in(0, \pi)$. Hence $a_{2} \neq a_{3}$ in the expression $y=\cot \theta_{2}$ and $y=\cot \theta_{3}$ because of the hypothesis $P(\theta, \varphi) Q(\theta, \varphi) \neq 0$. By checking, it is proved that $\theta_{1}, \theta_{2}$ are the roots of $\frac{\mathrm{d}(\sqrt{P(\theta, 0)}+\sqrt{Q(\theta, 0)})}{\mathrm{d} \theta}=0$. Thus

$$
\begin{aligned}
& \min \{\sqrt{P(\theta, 0)}+\sqrt{Q(\theta, 0)}\} \\
= & \begin{cases}\min \left\{\sqrt{P\left(\theta_{1}, 0\right)}+\sqrt{Q\left(\theta_{1}, 0\right)}, \sqrt{P\left(\theta_{2}, 0\right)}+\sqrt{Q\left(\theta_{2}, 0\right)}, \sqrt{P(0,0)}+\sqrt{Q(0,0)}\right\}, & \text { if } a_{2} \neq a_{3}, \\
\min \left\{\left.\sqrt{P\left(\pi-\theta_{0}, 0\right)}\right|_{a_{2}=a_{3}}, \sqrt{P\left(\theta_{1}, 0\right)}+\sqrt{Q\left(\theta_{1}, 0\right)}, \sqrt{P(0,0)}+\sqrt{Q(0,0)}\right\}, & \text { if } a_{2}=a_{3} .\end{cases}
\end{aligned}
$$

For the cases $\varphi=\pi$ and $\varphi=2 \pi$, from eqs. (19)-(21), we have

$$
\begin{aligned}
\min \{\sqrt{P(\theta, \pi)}+\sqrt{Q(\theta, \pi)}\} & =\min \{\sqrt{P(\pi-\theta, 0)}+\sqrt{Q(\pi-\theta, 0)}\} \\
& =\min \{\sqrt{P(\theta, 0)}+\sqrt{Q(\theta, 0)}\} \\
& =\min \{\sqrt{P(\theta, 2 \pi)}+\sqrt{Q(\theta, 2 \pi)}\} .
\end{aligned}
$$

Therefore, if $a_{2} \neq a_{3}$, then

$$
\begin{aligned}
& \min \{\sqrt{P(\theta, \varphi)}+\sqrt{Q(\theta, \varphi)}\} \\
& =\min \left\{\sqrt{P(0, \varphi)}+\sqrt{Q(0, \varphi)}, \sqrt{P\left(\theta_{1}, 0\right)}+\sqrt{Q\left(\theta_{1}, 0\right)}, \sqrt{P\left(\theta_{2}, 0\right)}\right. \\
& \left.+\sqrt{Q\left(\theta_{2}, 0\right)}, \sqrt{P\left(\frac{\pi}{2}, \frac{\pi}{2}\right)}+\sqrt{Q\left(\frac{\pi}{2}, \frac{\pi}{2}\right)}\right\} ;
\end{aligned}
$$

if $a_{2}=a_{3}$, then

$$
\begin{aligned}
& \min \{\sqrt{P(\theta, \varphi)}+\sqrt{Q(\theta, \varphi)}\} \\
= & \min \left\{\sqrt{P(0, \varphi)}+\sqrt{Q(0, \varphi)},\left.\sqrt{P\left(\pi-\theta_{0}, 0\right)}\right|_{a_{2}=a_{3},}\right. \\
& \left.\sqrt{P\left(\theta_{1}, 0\right)}+\sqrt{Q\left(\theta_{1}, 0\right)}, \sqrt{P\left(\frac{\pi}{2}, \frac{\pi}{2}\right)}+\sqrt{Q\left(\frac{\pi}{2}, \frac{\pi}{2}\right)}\right\} .
\end{aligned}
$$

\section{$2.7 \quad a_{4}=0$ and $a_{0} a_{1} a_{2} a_{3} \neq 0$}

In this section, we consider the quantum channel (2) with coefficients satisfying $a_{4}=0$ and $a_{0} a_{1} a_{2} a_{3} \neq 0$.

Note that

$$
\begin{aligned}
\sqrt{P(0, \varphi)}+\sqrt{Q(0, \varphi)} & =\sqrt{P(\pi, \varphi)}+\sqrt{Q(\pi, \varphi)}=a_{0}^{2}+\sqrt{\left(1-a_{0}^{2}\right)^{2}-4 a_{2}^{2} a_{3}^{2}} \\
& =a_{0}^{2}+\sqrt{\left[a_{1}^{2}+\left(a_{2}-a_{3}\right)^{2}\right]\left[a_{1}^{2}+\left(a_{2}+a_{3}\right)^{2}\right]}
\end{aligned}
$$


and $P(\theta, \varphi) Q(\theta, \varphi) \neq 0$ in the case of $\theta=0, \pi$. Next we suppose that $\sin \theta \neq 0$, that is $\theta \in(0, \pi)$.

We begin with the discussion of the condition satisfying $P(\theta, \varphi)=0$. We see that

$$
\begin{aligned}
P(\theta, \varphi)= & \frac{1}{4}\left[1+2 a_{0} a_{1} \cos (\varphi-\mu) \sin \theta-2 a_{2} a_{3}-\cos \theta\left(1-2 a_{0}^{2}-2 a_{2} a_{3}\right)\right] \\
& \times\left[1+2 a_{0} a_{1} \cos (\varphi-\mu) \sin \theta+2 a_{2} a_{3}-\cos \theta\left(1-2 a_{0}^{2}+2 a_{2} a_{3}\right)\right] \\
= & \frac{1}{\left(1+t^{2}\right)^{2}}\left[1+\left(t^{2}-1\right) a_{0}^{2}+2 t a_{0} a_{1} \cos (\varphi-\mu)-2 a_{2} a_{3}\right] \\
& \times\left[1+\left(t^{2}-1\right) a_{0}^{2}+2 t a_{0} a_{1} \cos (\varphi-\mu)+2 a_{2} a_{3}\right] \\
= & 0,
\end{aligned}
$$

which implies that

$$
\cos (\varphi-\mu)=z_{1}=\frac{-1+a_{0}^{2}-t^{2} a_{0}^{2}-2 a_{2} a_{3}}{2 t a_{0} a_{1}}, \quad \cos (\varphi-\mu)=z_{2}=\frac{-1+a_{0}^{2}-t^{2} a_{0}^{2}+2 a_{2} a_{3}}{2 t a_{0} a_{1}} .
$$

Note that both $z_{1}=\frac{-1+a_{0}^{2}-t^{2} a_{0}^{2}-2 a_{2} a_{3}}{2 t a_{0} a_{1}}$ and $z_{2}=\frac{-1+a_{0}^{2}-t^{2} a_{0}^{2}+2 a_{2} a_{3}}{2 t a_{0} a_{1}}$ tend to $-\infty$ when $t \rightarrow 0$ and $t \rightarrow+\infty$. Because

$$
z_{1} \leqslant-2 \sqrt{\frac{1-a_{0}^{2}+2 a_{2} a_{3}}{2 t a_{0} a_{1}}} \sqrt{\frac{t^{2} a_{0}^{2}}{2 t a_{0} a_{1}}}=-\frac{\sqrt{a_{1}^{2}+\left(a_{2}+a_{3}\right)^{2}}}{a_{1}}<-1,
$$

there is no $\varphi$ such that $\cos (\varphi-\mu)=z_{1}=\frac{-1+a_{0}^{2}-t^{2} a_{0}^{2}-2 a_{2} a_{3}}{2 t a_{0} a_{1}}$. Note that

$$
z_{2} \leqslant-2 \sqrt{\frac{1-a_{0}^{2}-2 a_{2} a_{3}}{2 t a_{0} a_{1}}} \sqrt{\frac{t^{2} a_{0}^{2}}{2 t a_{0} a_{1}}}=-\frac{\sqrt{a_{1}^{2}+\left(a_{2}-a_{3}\right)^{2}}}{a_{1}} \leqslant-1,
$$

where the equality $z_{2}=-1$ holds iff $t=\frac{a_{1}}{a_{0}}$ and $a_{2}=a_{3}$. It follows that

$$
\cos (\varphi-\mu)=z_{2}=\frac{-1+a_{0}^{2}-t^{2} a_{0}^{2}+2 a_{2} a_{3}}{2 t a_{0} a_{1}}
$$

iff $a_{2}=a_{3}, \varphi=\pi+\mu$, and $\theta=\theta_{0}$, where $\cot \frac{\theta_{0}}{2}=\frac{a_{1}}{a_{0}}$. Thus, $P(\theta, \varphi)=0$ if and only if $a_{2}=a_{3}, \varphi=\pi+\mu$, and $\theta=\theta_{0}$. Therefore,

$$
\begin{aligned}
\left.(\sqrt{P(\theta, \varphi)}+\sqrt{Q(\theta, \varphi)})\right|_{P(\theta, \varphi)=0} & =\left.\sqrt{Q\left(\theta_{0}, \mu+\pi\right)}\right|_{a_{2}=a_{3}} \\
& =\frac{\sqrt{\left(a_{0}^{4}+a_{1}^{2}+a_{0}^{2} a_{1}^{2}-2 a_{1}^{2} a_{3}^{2}\right)\left(a_{0}^{4}+a_{1}^{2}+a_{0}^{2} a_{1}^{2}+2 a_{1}^{2} a_{3}^{2}\right)}}{a_{0}^{2}+a_{1}^{2}} .
\end{aligned}
$$

By eq. (19), we know that $Q(\theta, \varphi)=0$ if and only if $a_{2}=a_{3}, \varphi=\mu$, and $\theta=\pi-\theta_{0}$ and

$$
\left.(\sqrt{P(\theta, \varphi)}+\sqrt{Q(\theta, \varphi)})\right|_{Q(\theta, \varphi)=0}=\left.\sqrt{P\left(\pi-\theta_{0}, \mu\right)}\right|_{a_{2}=a_{3}}
$$




$$
=\left.(\sqrt{P(\theta, \varphi)}+\sqrt{Q(\theta, \varphi)})\right|_{P(\theta, \varphi)=0} .
$$

From above, we see that the quantum channel with $a_{4}=0, a_{2}=a_{3}$, and $a_{0} a_{1} a_{2} a_{3} \neq 0$ is collapsed to an EPR pair with probability $p_{1}=p_{2}=\frac{a_{0}^{2}\left(1-a_{0}^{2}-a_{1}^{2}\right)}{a_{0}^{2}+a_{1}^{2}}$ via Charlie's appropriate measurement (Charlie measures his particle in the measurement basis (3) with $(\theta, \varphi)=\left(\theta_{0}, \mu+\pi\right)$ or $\left.(\theta, \varphi)=\left(\pi-\theta_{0}, \mu\right)\right)$.

In the following, we suppose that $P(\theta, \varphi) Q(\theta, \varphi) \neq 0$. From eqs. (26) and (27), we get

$$
\begin{aligned}
& \sin (\varphi-\mu)\left[a_{0} \cos \theta+a_{1} \cos (\varphi-\mu) \sin \theta\right]=0, \\
& \sin (\varphi-\mu)\left[a_{0} \cos \theta+a_{1} \cos (\varphi-\mu) \sin \theta\right] \\
\times & {\left[\sin ^{2} \theta+2 a_{0}^{2} \cos ^{2} \theta+2 a_{0} a_{1} \cos \theta \cos (\varphi-\mu) \sin \theta\right]=0 . }
\end{aligned}
$$

It follows that the minimum of $\sqrt{P(\theta, \varphi)}+\sqrt{Q(\theta, \varphi)}$ must occur at either the hyperplane $\sin (\varphi-\mu)=0$ or the hyperplane $a_{0} \cos \theta+a_{1} \cos (\varphi-\mu) \sin \theta=0$ when $P(\theta, \varphi) Q(\theta, \varphi)$ $\neq 0$ and $\theta \in(0, \pi)$. Now we consider the two hyperplanes.

If $a_{0} \cos \theta+a_{1} \cos (\varphi-\mu) \sin \theta=0$, then $\cos (\varphi-\mu)=-\frac{a_{0} \cot \theta}{a_{1}}$,

$$
\left.(\sqrt{P(\theta, \varphi)}+\sqrt{Q(\theta, \varphi)})\right|_{\cos (\varphi-\mu)=-\frac{a_{0} \cot \theta}{a_{1}}}=\sqrt{1-4 a_{2}^{2} a_{3}^{2}} .
$$

For $\sin (\varphi-\mu)=0$, i.e. $\varphi=\mu, \mu+\pi$ in the case of $\mu \in(0, \pi]$, or $\varphi=0, \pi, 2 \pi$ in the case of $\mu=0$, we first investigate the case $\varphi=\mu$. From eq. (28), we have

$$
\begin{aligned}
& \left(a_{0} \cos \theta+a_{1} \sin \theta\right)\left[2 a_{0} a_{1}\left(a_{0}^{2}-a_{1}^{2}\right) \sin 2 \theta\right. \\
& \left.\quad-2 a_{0}^{2}\left(\sin ^{2} \theta+2 a_{1}^{2} \cos 2 \theta\right)+\sin ^{2} \theta\left(1-2 a_{1}^{2}-4 a_{2}^{2} a_{3}^{2}\right)\right]=0,
\end{aligned}
$$

that is, $a_{0} \cos \theta+a_{1} \sin \theta=0$, or

$$
2 a_{0} a_{1}\left(a_{0}^{2}-a_{1}^{2}\right) \sin 2 \theta-2 a_{0}^{2}\left(\sin ^{2} \theta+2 a_{1}^{2} \cos 2 \theta\right)+\sin ^{2} \theta\left(1-2 a_{1}^{2}-4 a_{2}^{2} a_{3}^{2}\right)=0 .
$$

From $a_{0} \cos \theta+a_{1} \sin \theta=0$, there is

$$
\cot \theta_{1}=-\frac{a_{1}}{a_{0}}
$$

Thus, $\theta_{1}$ is a root of eq. (24). From

$$
2 a_{0} a_{1}\left(a_{0}^{2}-a_{1}^{2}\right) \sin 2 \theta-2 a_{0}^{2}\left(\sin ^{2} \theta+2 a_{1}^{2} \cos 2 \theta\right)+\sin ^{2} \theta\left(1-2 a_{1}^{2}-4 a_{2}^{2} a_{3}^{2}\right)=0,
$$

there is

$$
\cot \theta_{2}=\frac{a_{0}^{2}-a_{1}^{2}-\left|a_{2}^{2}-a_{3}^{2}\right|}{2 a_{0} a_{1}}, \quad \cot \theta_{3}=\frac{a_{0}^{2}-a_{1}^{2}+\left|a_{2}^{2}-a_{3}^{2}\right|}{2 a_{0} a_{1}},
$$

where $a_{2} \neq a_{3}$ (because when $a_{2}=a_{3}$ there is $\theta_{2}=\theta_{3}=\pi-\theta_{0}$, i.e. $Q\left(\pi-\theta_{0}, \mu\right)=0$, which contradicts with the hypothesis $P(\theta, \varphi) Q(\theta, \varphi) \neq 0)$. By checking, we see $\theta_{3}$ is a root of $\frac{\partial(\sqrt{P(\theta, \mu)}+\sqrt{Q(\theta, \mu)})}{\partial \theta}=0$, while $\theta_{2}$ is not. Hence, 


$$
\begin{aligned}
& \min \{\sqrt{P(\theta, \mu)}+\sqrt{Q(\theta, \mu)}\} \\
& =\left\{\begin{array}{c}
\min \left\{\sqrt{P\left(\theta_{1}, \mu\right)}+\sqrt{Q\left(\theta_{1}, \mu\right)}, \sqrt{P\left(\theta_{3}, \mu\right)}+\sqrt{Q\left(\theta_{3}, \mu\right)}, \sqrt{P(0, \mu)}+\sqrt{Q(0, \mu)}\right\}, \\
\text { if } a_{2} \neq a_{3}, \\
\min \left\{\sqrt{P\left(\theta_{1}, \mu\right)}+\sqrt{Q\left(\theta_{1}, \mu\right)},\left.\sqrt{P\left(\pi-\theta_{0}, \mu\right)}\right|_{a_{2}=a_{3}}, \sqrt{P(0, \mu)}+\sqrt{Q(0, \mu)}\right\}, \\
\text { if } a_{2}=a_{3} .
\end{array}\right.
\end{aligned}
$$

For $\varphi=\mu+\pi, \mu+2 \pi$, from eqs. (19) and (20) there is

$$
\begin{aligned}
\min \{\sqrt{P(\theta, \mu+\pi)}+\sqrt{Q(\theta, \mu+\pi)}\} & =\min \{\sqrt{P(\pi-\theta, \mu)}+\sqrt{Q(\pi-\theta, \mu)}\} \\
& =\min \{\sqrt{P(\theta, \mu)}+\sqrt{Q(\theta, \mu)}\} \\
& =\min \{\sqrt{P(\theta, \mu+2 \pi)}+\sqrt{Q(\theta, \mu+2 \pi)}\} .
\end{aligned}
$$

Thus, if $a_{2}=a_{3}$, then

$$
\begin{aligned}
& \min \{\sqrt{P(\theta, \varphi)}+\sqrt{Q(\theta, \varphi)}\} \\
= & \min \left\{a_{0}^{2}+\sqrt{\left(1-a_{0}^{2}\right)^{2}-4 a_{2}^{2} a_{3}^{2}}, \sqrt{1-4 a_{2}^{2} a_{3}^{2}}, \sqrt{P\left(\theta_{1}, \mu\right)}+\sqrt{Q\left(\theta_{1}, \mu\right)},\left.\sqrt{Q\left(\theta_{0}, \pi+\mu\right)}\right|_{a_{2}=a_{3}}\right\} ;
\end{aligned}
$$

if $a_{2} \neq a_{3}$, then

$$
\begin{aligned}
& \min \{\sqrt{P(\theta, \varphi)}+\sqrt{Q(\theta, \varphi)}\} \\
= & \min \left\{a_{0}^{2}+\sqrt{\left(1-a_{0}^{2}\right)^{2}-4 a_{2}^{2} a_{3}^{2}}, \sqrt{1-4 a_{2}^{2} a_{3}^{2}}, \sqrt{P\left(\theta_{1}, \mu\right)}+\sqrt{Q\left(\theta_{1}, \mu\right)}, \sqrt{P\left(\theta_{3}, \mu\right)}+\sqrt{Q\left(\theta_{3}, \mu\right)}\right\} .
\end{aligned}
$$

Here, $\cot \frac{\theta_{0}}{2}=\frac{a_{1}}{a_{0}}, \quad \cot \theta_{1}=-\frac{a_{1}}{a_{0}}, \quad \cot \theta_{3}=\frac{a_{0}^{2}-a_{1}^{2}+\left|a_{2}^{2}-a_{3}^{2}\right|}{2 a_{0} a_{1}}$.

\subsection{One is $a_{2}=0$ and $a_{0} a_{1} a_{3} a_{4} \neq 0$; the other is $a_{3}=0$ and $a_{0} a_{1} a_{2} a_{4} \neq 0$}

We now discuss the quantum channel (2), the coefficients of which satisfy $a_{2}=0$, but $a_{0} a_{1} a_{3} a_{4} \neq 0$.

When $\sin \theta=0$, i.e. $\theta=0, \pi$, then

$$
\begin{aligned}
\sqrt{P(0, \varphi)}+\sqrt{Q(0, \varphi)} & =\sqrt{P(\pi, \varphi)}+\sqrt{Q(\pi, \varphi)} \\
& =a_{0}^{2}+\sqrt{\left(1-a_{0}^{2}\right)^{2}-4 a_{1}^{2} a_{4}^{2}} \\
& =a_{0}^{2}+\sqrt{\left[a_{3}^{2}+\left(a_{1}-a_{4}\right)^{2}\right]\left[a_{3}^{2}+\left(a_{1}+a_{4}\right)^{2}\right]},
\end{aligned}
$$

and $P(\theta, \varphi) Q(\theta, \varphi) \neq 0$ in the case of $\theta=0, \pi$. Next we suppose that $\sin \theta \neq 0$.

First, we prove that $P(\theta, \varphi) Q(\theta, \varphi) \neq 0$. If $P(\theta, \varphi)=0$, then

$$
\cos (\varphi-\mu)=\frac{-a_{0}^{3} a_{1} \sin 2 \theta+8 \sqrt{-a_{0}^{2} a_{1}^{2} a_{3}^{2} a_{4}^{2} \cos ^{2} \frac{\theta}{2} \sin ^{6} \frac{\theta}{2}}-4 a_{0} a_{1}\left(1-2 a_{4}^{2}\right) \cos \frac{\theta}{2} \sin ^{3} \frac{\theta}{2}}{2 a_{0}^{2} a_{1}^{2} \sin ^{2} \theta},
$$




$$
\cos (\varphi-\mu)=\frac{a_{0}^{3} a_{1} \sin 2 \theta+8 \sqrt{-a_{0}^{2} a_{1}^{2} a_{3}^{2} a_{4}^{2} \cos ^{2} \frac{\theta}{2} \sin ^{6} \frac{\theta}{2}}+4 a_{0} a_{1}\left(1-2 a_{4}^{2}\right) \cos \frac{\theta}{2} \sin ^{3} \frac{\theta}{2}}{-2 a_{0}^{2} a_{1}^{2} \sin ^{2} \theta} .
$$

But $8 \sqrt{-a_{0}^{2} a_{1}^{2} a_{3}^{2} a_{4}^{2} \cos ^{2} \frac{\theta}{2} \sin ^{6} \frac{\theta}{2}}$ is an imaginary number, so $P(\theta, \varphi) \neq 0$. From eq. (19), there is also $Q(\theta, \varphi) \neq 0$. That is, no matter what kind of measurement basis Charlie chooses, Alice and Bob can never share an EPR pair after his measurement.

Second, the minimum of $\sqrt{P(\theta, \varphi)}+\sqrt{Q(\theta, \varphi)}$ should occur at the point satisfying eqs. (24) and (25), or $\theta=0$. From eqs. (26) and (27), there are

$$
\sin (\varphi-\mu)\left[a_{0} \cos \theta+a_{1} \cos (\varphi-\mu) \sin \theta\right]=0,
$$

and

$$
\begin{aligned}
& \sin (\varphi-\mu)\left[a_{0} \cos \theta+a_{1} \cos (\varphi-\mu) \sin \theta\right] \\
& \quad \times\left[2 a_{0}^{2} \cos ^{2} \theta+a_{0} a_{1} \cos (\varphi-\mu) \sin 2 \theta+\left(1-2 a_{4}^{2}\right) \sin ^{2} \theta\right]=0 .
\end{aligned}
$$

It follows that the minimum of $\sqrt{P(\theta, \varphi)}+\sqrt{Q(\theta, \varphi)}$ should occur at the point such that $\sin (\varphi-\mu)=0$, or $a_{0} \cos \theta+a_{1} \cos (\varphi-\mu) \sin \theta=0$, or $\theta=0$.

From $a_{0} \cos \theta+a_{1} \cos (\varphi-\mu) \sin \theta=0$, we know that $\cos (\varphi-\mu)=-\frac{a_{0} \cot \theta}{a_{1}}$, and

$$
\left.(\sqrt{P(\theta, \varphi)}+\sqrt{Q(\theta, \varphi)})\right|_{\left\{\theta \in(0, \pi), \cos (\varphi-\mu)=-\frac{a_{0} \cot \theta}{a_{1}}\right\}}=\sqrt{1-4 a_{0}^{2} a_{4}^{2}-4 a_{1}^{2} a_{4}^{2}} .
$$

For the case $\sin (\varphi-\mu)=0$, since eq. (64) holds, we only need to consider the case $\varphi=\mu$. From eq. (28), we derive

$$
\begin{aligned}
& \sin 3 \theta\left(2 a_{0}^{2} a_{1}-a_{1}+4 a_{0}^{4} a_{1}+2 a_{1}^{3}-12 a_{0}^{2} a_{1}^{3}\right)+\sin \theta\left(3 a_{1}-6 a_{0}^{2} a_{1}+4 a_{0}^{4} a_{1}-6 a_{1}^{3}+4 a_{0}^{2} a_{1}^{3}\right) \\
& +\cos \theta\left(a_{0}-2 a_{0}^{3}-2 a_{0} a_{1}^{2}-4 a_{0}^{3} a_{1}^{2}-4 a_{0} a_{1}^{4}\right)+\cos 3 \theta\left(2 a_{0}^{3}-a_{0}+2 a_{0} a_{1}^{2}-12 a_{0}^{3} a_{1}^{2}+4 a_{0} a_{1}^{4}\right) \\
= & \left\{-16 \cot ^{3} \theta a_{0}^{3} a_{1}^{2}+16 \cot ^{2} \theta a_{0}^{2} a_{1}\left(a_{0}^{2}-2 a_{1}^{2}\right)+4 \cot \theta a_{0}\left[1-2 a_{1}^{2}-4 a_{1}^{4}+2 a_{0}^{2}\left(4 a_{1}^{2}-1\right)\right]\right. \\
& \left.+4\left(1-2 a_{0}^{2}\right) a_{1}\left(1-2 a_{1}^{2}\right)\right\} \sin ^{3} \theta \\
= & 0,
\end{aligned}
$$

which follows that

$$
\cot \theta=\cot \theta_{1}=-\frac{a_{1}}{a_{0}}, \quad \cot \theta=\cot \theta_{2}=\frac{1-2 a_{1}^{2}}{2 a_{0} a_{1}}, \quad \cot \theta=\cot \theta_{3}=\frac{-1+2 a_{0}^{2}}{2 a_{0} a_{1}},
$$

where $\theta_{1}$ and $\theta_{2}$ are the roots of equation $\frac{\partial(\sqrt{P(\theta, \mu)}+\sqrt{Q(\theta, \mu)})}{\partial \theta}=0$, while $\theta_{3}$ is not. It is not difficult to obtain

$$
\begin{aligned}
& \min \{\sqrt{P(\theta, \mu)}+\sqrt{Q(\theta, \mu)}\} \\
= & \min \left\{\sqrt{P\left(\theta_{1}, \mu\right)}+\sqrt{Q\left(\theta_{1}, \mu\right)}, \sqrt{P\left(\theta_{2}, \mu\right)}+\sqrt{Q\left(\theta_{2}, \mu\right)}, \sqrt{P(0, \mu)}+\sqrt{Q(0, \mu)}\right\} .
\end{aligned}
$$

Therefore, for the quantum channel (2) with $a_{2}=0$ and $a_{0} a_{1} a_{3} a_{4} \neq 0$, there is 


$$
\begin{aligned}
& \min \{\sqrt{P(\theta, \varphi)}+\sqrt{Q(\theta, \varphi)}\} \\
= & \min \left\{\sqrt{P\left(\theta_{1}, \mu\right)}+\sqrt{Q\left(\theta_{1}, \mu\right)}, \sqrt{P\left(\theta_{2}, \mu\right)}+\sqrt{Q\left(\theta_{2}, \mu\right)},\right. \\
& \left.\sqrt{P(0, \varphi)}+\sqrt{Q(0, \varphi)}, \sqrt{1-4 a_{0}^{2} a_{4}^{2}-4 a_{1}^{2} a_{4}^{2}}\right\} .
\end{aligned}
$$

Similarly, for the quantum channel (2) with $a_{3}=0$ and $a_{0} a_{1} a_{2} a_{4} \neq 0$ we also obtain

$$
\begin{aligned}
& \min \{\sqrt{P(\theta, \varphi)}+\sqrt{Q(\theta, \varphi)}\} \\
= & \min \left\{\sqrt{P\left(\theta_{1}, \mu\right)}+\sqrt{Q\left(\theta_{1}, \mu\right)}, \sqrt{P\left(\theta_{2}, \mu\right)}+\sqrt{Q\left(\theta_{2}, \mu\right)},\right. \\
& \left.a_{0}^{2}+\sqrt{\left(1-a_{0}^{2}\right)^{2}-4 a_{1}^{2} a_{4}^{2}}, \sqrt{1-4 a_{0}^{2} a_{4}^{2}-4 a_{1}^{2} a_{4}^{2}}\right\} .
\end{aligned}
$$

Here $\cot \theta_{1}=-\frac{a_{1}}{a_{0}}$.

\section{$2.9 \mu=0$ and $a_{0} a_{1} a_{2} a_{3} a_{4} \neq 0$}

In this section, we investigate the quantum channel (2) with $\mu=0$ and $a_{0} a_{1} a_{2} a_{3} a_{4} \neq 0$. It is seen that

$$
\begin{aligned}
\sqrt{P(0, \varphi)}+\sqrt{Q(0, \varphi)} & =\sqrt{P(\pi, \varphi)}+\sqrt{Q(\pi, \varphi)} \\
& =a_{0}^{2}+\sqrt{\left(1-a_{0}^{2}\right)^{2}-4\left(a_{2} a_{3}-a_{1} a_{4}\right)^{2}} \\
& =a_{0}^{2}+\sqrt{\left[\left(a_{2}-a_{3}\right)^{2}+\left(a_{1}+a_{4}\right)^{2}\right]\left[\left(a_{2}+a_{3}\right)^{2}+\left(a_{1}-a_{4}\right)^{2}\right]},
\end{aligned}
$$

and $P(\theta, \varphi) Q(\theta, \varphi) \neq 0$ in the case of $\theta=0, \pi$. In the following, we suppose that $\theta \neq 0$, $\pi$, i.e. $\theta \in(0, \pi)$.

We first consider the case $P(\theta, \varphi)=0$. If $P(\theta, \varphi)=0$, then

$$
\begin{aligned}
\cos \varphi= & z_{1} \\
= & \frac{1}{2 t a_{0} a_{1}^{2}}\left\{-t^{2} a_{0}^{2} a_{1}-\left(1-a_{0}^{2}\right) a_{1}-2\left(a_{2} a_{3}-a_{1} a_{4}\right) a_{4}\right. \\
& \left.-2\left[t^{2} a_{0}^{2} a_{1} a_{2} a_{3} a_{4}+\left(a_{2} a_{3}-a_{1} a_{4}\right)\left(a_{1} a_{3}+a_{2} a_{4}\right)\left(a_{1} a_{2}+a_{3} a_{4}\right)\right]^{\frac{1}{2}}\right\}, \\
\cos \varphi= & z_{2} \\
= & \frac{1}{2 t a_{0} a_{1}^{2}}\left\{-t^{2} a_{0}^{2} a_{1}-\left(1-a_{0}^{2}\right) a_{1}-2\left(a_{2} a_{3}-a_{1} a_{4}\right) a_{4}\right. \\
& \left.+2\left[t^{2} a_{0}^{2} a_{1} a_{2} a_{3} a_{4}+\left(a_{2} a_{3}-a_{1} a_{4}\right)\left(a_{1} a_{3}+a_{2} a_{4}\right)\left(a_{1} a_{2}+a_{3} a_{4}\right)\right]^{\frac{1}{2}}\right\} .
\end{aligned}
$$

Note that if $a_{1} a_{4}-a_{2} a_{3}>0$, then $t \in\left[t_{0},+\infty\right)$; if $a_{1} a_{4}-a_{2} a_{3} \leqslant 0$, then $t \in(0,+\infty)$. Here, $t_{0}=$ $\sqrt{\frac{\left(a_{1} a_{4}-a_{2} a_{3}\right)\left(a_{1} a_{3}+a_{2} a_{4}\right)\left(a_{1} a_{2}+a_{3} a_{4}\right)}{a_{0}^{2} a_{1} a_{2} a_{3} a_{4}}}$. We see that both $z_{1}$ and $z_{2}$ go to $-\infty$ when $t \rightarrow+\infty$. 
It is proved that

$$
\begin{aligned}
z= & z_{1}=\frac{1}{2 t a_{0} a_{1}^{2}}\left\{-t^{2} a_{0}^{2} a_{1}-\left(1-a_{0}^{2}\right) a_{1}-2\left(a_{2} a_{3}-a_{1} a_{4}\right) a_{4}\right. \\
& \left.-2\left[t^{2} a_{0}^{2} a_{1} a_{2} a_{3} a_{4}+\left(a_{2} a_{3}-a_{1} a_{4}\right)\left(a_{1} a_{3}+a_{2} a_{4}\right)\left(a_{1} a_{2}+a_{3} a_{4}\right)\right]^{\frac{1}{2}}\right\}
\end{aligned}
$$

has no intersection point with the straight line $z=-1 . \quad z_{1}$ is a continuous function of $t$ and tends to $-\infty$ when $t$ tends to $+\infty$, so $z_{1}<-1$. Thus, there is no $\varphi$ satisfying $\cos \varphi=z_{1}$. Now we look at $z=z_{2} . \quad z_{2}=-1$ implies that

$$
\begin{aligned}
& {\left[-t^{2} a_{0}^{2} a_{1}-\left(1-a_{0}^{2}\right) a_{1}-2\left(a_{2} a_{3}-a_{1} a_{4}\right) a_{4}+2 t a_{0} a_{1}^{2}\right]^{2} } \\
& -4\left\{t^{2} a_{0}^{2} a_{1} a_{2} a_{3} a_{4}+\left(a_{2} a_{3}-a_{1} a_{4}\right)\left(a_{1} a_{3}+a_{2} a_{4}\right)\left(a_{1} a_{2}+a_{3} a_{4}\right)\right\} \\
= & a_{1}^{2}\left[-t^{2} a_{0}^{2}+2 t a_{0}\left(a_{1}+a_{4}\right)-1+a_{0}^{2}+2 a_{2} a_{3}-2 a_{1} a_{4}\right] \\
\times & {\left[-t^{2} a_{0}^{2}+2 t a_{0}\left(a_{1}-a_{4}\right)-1+a_{0}^{2}-2 a_{2} a_{3}+2 a_{1} a_{4}\right] } \\
= & 0,
\end{aligned}
$$

which follows that

$$
\begin{aligned}
& t=t_{1}=\frac{a_{1}-a_{4}-\sqrt{-\left(a_{2}+a_{3}\right)^{2}}}{a_{0}}, \quad t=t_{2}=\frac{a_{1}-a_{4}+\sqrt{-\left(a_{2}+a_{3}\right)^{2}}}{a_{0}}, \\
& t=t_{3}=\frac{a_{1}+a_{4}-\sqrt{-\left(a_{2}-a_{3}\right)^{2}}}{a_{0}}, \quad t=t_{4}=\frac{a_{1}+a_{4}+\sqrt{-\left(a_{2}-a_{3}\right)^{2}}}{a_{0}},
\end{aligned}
$$

where $t_{1}$ and $t_{2}$ are imaginary numbers, while $t_{3}$ and $t_{4}$ are real numbers only if $a_{2}=a_{3}$. By checking, when $a_{2}=a_{3}, t_{3}=t_{4}=\frac{a_{1}+a_{4}}{a_{0}}$ is a root of equation $z_{2}=-1$, and the maximum point of function $z=z_{2}$. Because $z=z_{2}$ is a continuous function of $t$ and tends to $-\infty$ when $t$ tends to $+\infty$, and has only one intersection point with the straight line $z=-1$, there must be $z_{2} \leqslant-1$, where the equality holds iff $a_{2}=a_{3}$ and $t=t_{3}=t_{4}=\frac{a_{1}+a_{4}}{a_{0}}$. It means that $P(\theta, \varphi)=0$ iff $a_{2}=a_{3}, \varphi=\pi$, and $\theta=\theta_{0}$, where $\cot \frac{\theta_{0}}{2}=\frac{a_{1}+a_{4}}{a_{0}}$. From eq. (19), we show that $Q(\theta, \varphi)=0$ iff $a_{2}=a_{3}, \varphi=0$, and $\theta=\pi-\theta_{0}$. Therefore,

$$
\begin{aligned}
\left.(\sqrt{P(\theta, \varphi)}+\sqrt{Q(\theta, \varphi)})\right|_{P(\theta, \varphi)=0} & =\left.\sqrt{Q\left(\theta_{0}, \pi\right)}\right|_{a_{2}=a_{3}} \\
& =\left.\sqrt{P\left(\pi-\theta_{0}, 0\right)}\right|_{a_{2}=a_{3}} \\
& =\left.(\sqrt{P(\theta, \varphi)}+\sqrt{Q(\theta, \varphi)})\right|_{Q(\theta, \varphi)=0} \\
& =\sqrt{\left(1-2 a_{4}^{2}\right)^{2}+4 a_{3}^{2}\left(-1+a_{1}^{2}+a_{3}^{2}+2 a_{1} a_{4}+3 a_{4}^{2}\right)} .
\end{aligned}
$$

It is shown that the quantum channel (2) with $\mu=0, a_{0} a_{1} a_{2} a_{3} a_{4} \neq 0$ and $a_{2}=a_{3}$ collapses to an EPR pair with probability $p_{1}=p_{2}=\frac{a_{0}^{2}\left(1-a_{0}^{2}+3 a_{1}^{2}+4 a_{1} a_{4}+a_{4}^{2}\right)}{a_{0}^{2}+\left(a_{1}+a_{4}\right)^{2}}$ after Charlie's measure- 
ment in the basis (3) with either $\varphi=\pi$ and $\theta=\theta_{0}$, or $\varphi=0$ and $\theta=\pi-\theta_{0}$, in the case of $a_{2}=a_{3}$.

In the following we suppose that $P(\theta, \varphi) Q(\theta, \varphi) \neq 0$. By eq. (26), there is

$$
\begin{aligned}
& \sin \varphi\left\{2 \cos \varphi\left(a_{2} a_{3}-a_{1} a_{4}\right)\left(a_{1} a_{3}+a_{2} a_{4}\right)\left(a_{1} a_{2}+a_{3} a_{4}\right)-y a_{0}\left[a_{2} a_{3} a_{4}\left(1+2 a_{1}^{2}-2 a_{2}^{2}-2 a_{3}^{2}\right)\right.\right. \\
& \left.\left.-2 a_{1}\left(a_{2}^{2} a_{3}^{2}-a_{2}^{2} a_{4}^{2}-a_{3}^{2} a_{4}^{2}\right)\right]\right\}=0 .
\end{aligned}
$$

Note that

$$
a_{2} a_{3} a_{4}\left(1+2 a_{1}^{2}-2 a_{2}^{2}-2 a_{3}^{2}\right)-2 a_{1}\left(a_{2}^{2} a_{3}^{2}-a_{2}^{2} a_{4}^{2}-a_{3}^{2} a_{4}^{2}\right)=a_{1} a_{4}^{2} \neq 0,
$$

if $a_{2} a_{3}=a_{1} a_{4}$. Therefore,

$$
a_{2} a_{3} a_{4}\left(1+2 a_{1}^{2}-2 a_{2}^{2}-2 a_{3}^{2}\right)-2 a_{1}\left(a_{2}^{2} a_{3}^{2}-a_{2}^{2} a_{4}^{2}-a_{3}^{2} a_{4}^{2}\right)=0
$$

implies that $\cos \varphi=0$. Thus, eq. (77) implies that $\sin \varphi=0, \cos \varphi=0$, or

$$
y=\frac{2 \cos \varphi\left(a_{2} a_{3}-a_{1} a_{4}\right)\left[\left(a_{1}^{2}+a_{4}^{2}\right) a_{2} a_{3}+a_{1}\left(a_{2}^{2}+a_{3}^{2}\right) a_{4}\right]}{a_{0}\left[a_{2} a_{3} a_{4}\left(1+2 a_{1}^{2}-2 a_{2}^{2}-2 a_{3}^{2}\right)-2 a_{1}\left(a_{2}^{2} a_{3}^{2}-a_{2}^{2} a_{4}^{2}-a_{3}^{2} a_{4}^{2}\right)\right]},
$$

in the case of $\sin 2 \varphi \neq 0$.

From eq. (27), there is

$$
\begin{aligned}
& 4 y^{3} a_{0}^{3}\left(a_{2} a_{3}-a_{1} a_{4}\right)\left(a_{1} a_{3}+a_{2} a_{4}\right)\left(a_{1} a_{2}+a_{3} a_{4}\right) \\
& \quad+8 y^{2} a_{0}^{2} a_{1} \cos \varphi\left(a_{2} a_{3}-a_{1} a_{4}\right)\left(a_{1} a_{3}+a_{2} a_{4}\right)\left(a_{1} a_{2}+a_{3} a_{4}\right) \\
& \quad+y a_{0}\left\{4 a_{1}^{2} \cos ^{2} \varphi\left(a_{2} a_{3}-a_{1} a_{4}\right)\left(a_{1} a_{3}+a_{2} a_{4}\right)\left(a_{1} a_{2}+a_{3} a_{4}\right)\right. \\
& \left.\quad+\left(a_{1}+2 a_{2} a_{3} a_{4}-2 a_{1} a_{4}^{2}\right)\left[a_{2} a_{3}\left(-2 a_{4}^{3}-4 a_{1}^{2} a_{4}+a_{4}+2 a_{1} a_{2} a_{3}\right)-2 a_{1} a_{4}^{2}\left(a_{2}^{2}+a_{3}^{2}\right)\right]\right\} \\
& \quad+2 \cos \varphi\left(a_{1}+2 a_{2} a_{3} a_{4}-2 a_{1} a_{4}^{2}\right) \\
& \quad \times\left[a_{2}^{2} a_{3}^{2}\left(a_{1}^{2}+a_{4}^{2}\right)+a_{1} a_{2} a_{3} a_{4}\left(1-2 a_{1}^{2}-2 a_{4}^{2}\right)-a_{1}^{2} a_{4}^{2}\left(a_{2}^{2}+a_{3}^{2}\right)\right] \\
& =0 .
\end{aligned}
$$

Combining eqs. (78) and (79), we obtain that

$$
\begin{gathered}
\cos \varphi\left(a_{1}+2 a_{2} a_{3} a_{4}-2 a_{1} a_{4}^{2}\right)\left\{a_{0}^{2}\left[2 a_{1}\left(a_{2}^{2} a_{3}^{2}-a_{2}^{2} a_{4}^{2}-a_{3}^{2} a_{4}^{2}\right)-a_{2} a_{3} a_{4}\left(1+2 a_{1}^{2}-2 a_{2}^{2}-2 a_{3}^{2}\right)\right]^{2}\right. \\
\left.+4 \cos ^{2} \varphi\left(a_{2} a_{3}-a_{1} a_{4}\right)^{2}\left(a_{1} a_{3}+a_{2} a_{4}\right)^{2}\left(a_{1} a_{2}+a_{3} a_{4}\right)^{2}\right\}=0,
\end{gathered}
$$

which implies that $\cos \varphi=0$.

Next we only need to examine the two cases $\sin \varphi=0$ and $\cos \varphi=0$, that is, $\varphi=0, \frac{\pi}{2}, \pi, \frac{3 \pi}{2}, 2 \pi$.

Now look at the hyperplane $\varphi=0$. From eq. (28), we have

$$
\begin{aligned}
& {\left[2 y a_{0}\left(a_{2} a_{3}-a_{1} a_{4}\right)+2 a_{1} a_{2} a_{3}+a_{4}-2 a_{1}^{2} a_{4}\right]} \\
& \quad \times\left[a_{2} a_{3}-2 a_{0}^{2} a_{2} a_{3}-2 a_{1}^{2} a_{2} a_{3}+4 a_{0}^{2} a_{1}^{2} a_{2} a_{3}-4 a_{2}^{3} a_{3}^{3}-2 a_{1} a_{4}+6 a_{0}^{2} a_{1} a_{4}-4 a_{0}^{4} a_{1} a_{4}+2 a_{1}^{3} a_{4}\right. \\
& \quad-4 a_{0}^{2} a_{1}^{3} a_{4}+8 a_{1} a_{2}^{2} a_{3}^{2} a_{4}-2 a_{2} a_{3} a_{4}^{2}+4 a_{0}^{2} a_{2} a_{3} a_{4}^{2}-4 a_{1}^{2} a_{2} a_{3} a_{4}^{2}+2 a_{1} a_{4}^{3}-4 a_{0}^{2} a_{1} a_{4}^{3} \\
& \quad-4 y^{2} a_{0}^{2}\left(a_{1} a_{3}+a_{2} a_{4}\right)\left(a_{1} a_{2}+a_{3} a_{4}\right)-2 y a_{0}\left(-2 a_{0}^{2} a_{1} a_{2} a_{3}+2 a_{1}^{3} a_{2} a_{3}+a_{4}-3 a_{0}^{2} a_{4}\right. \\
& \left.\left.\quad+2 a_{0}^{4} a_{4}+a_{1}^{2} a_{4}-2 a_{1}^{4} a_{4}-4 a_{2}^{2} a_{3}^{2} a_{4}+6 a_{1} a_{2} a_{3} a_{4}^{2}-a_{4}^{3}+2 a_{0}^{2} a_{4}^{3}-2 a_{1}^{2} a_{4}^{3}\right)\right] \\
& =0 .
\end{aligned}
$$


First, we consider the case $a_{1} a_{4}-a_{2} a_{3} \neq 0$. In this case, eq. (81) has the following solution:

$$
y=\cot \theta_{1}=\frac{2 a_{1} a_{2} a_{3}+a_{4}-2 a_{1}^{2} a_{4}}{2 a_{0}\left(a_{1} a_{4}-a_{2} a_{3}\right)}
$$

where if $a_{2}=a_{3}$, then $a_{1} a_{4}-a_{2} a_{3} \neq \frac{1}{2}$;

$$
y=\cot \theta_{2}=\frac{-2 a_{1} a_{2} a_{4}+a_{3}\left(1-2 a_{1}^{2}-2 a_{3}^{2}-2 a_{4}^{2}\right)}{2 a_{0}\left(a_{1} a_{3}+a_{2} a_{4}\right)},
$$

where $a_{2} \neq a_{3}$;

$$
y=\cot \theta_{3}=\frac{-2 a_{1} a_{3} a_{4}+a_{2}\left(1-2 a_{1}^{2}-2 a_{2}^{2}-2 a_{4}^{2}\right)}{2 a_{0}\left(a_{1} a_{2}+a_{3} a_{4}\right)},
$$

where $a_{2} \neq a_{3}$.

By checking, we show that $\theta_{1}$ is a root of $\left.\frac{\mathrm{d}(\sqrt{P(\theta, 0)}+\sqrt{Q(\theta, 0)})}{\mathrm{d} \theta}\right|_{a_{1} a_{4}-a_{2} a_{3} \neq 0}=0$, while $\theta_{2}$ is a root only if $a_{2}>a_{3}$, and $\theta_{3}$ is a root only if $a_{2}<a_{3}$.

Second, we consider the case $a_{1} a_{4}-a_{2} a_{3}=0$. From eq. (81), we get

$$
y=\cot \theta_{4}=\frac{\left(1-2 a_{0}^{2}\right)\left(-1+a_{0}^{2}-a_{1}^{2}+a_{4}^{2}+\left|a_{2}^{2}-a_{3}^{2}\right|\right)}{4 a_{0}\left(1-a_{0}^{2}\right) a_{1}},
$$

where $a_{2} \neq a_{3}$;

$$
y=\cot \theta_{5}=\frac{\left(1-2 a_{0}^{2}\right)\left(-1+a_{0}^{2}-a_{1}^{2}+a_{4}^{2}-\left|a_{2}^{2}-a_{3}^{2}\right|\right)}{4 a_{0}\left(1-a_{0}^{2}\right) a_{1}},
$$

where $a_{2} \neq a_{3}$.

By checking, we know that only $\theta_{4}$ is a root of $\left.\frac{\mathrm{d}(\sqrt{P(\theta, 0)}+\sqrt{Q(\theta, 0)})}{\mathrm{d} \theta}\right|_{a_{1} a_{4}-a_{2} a_{3}=0}=0$.

Therefore,

$$
=\left\{\begin{array}{c}
\min \{\sqrt{P(\theta, 0)}+\sqrt{Q(\theta, 0)}\} \\
\min \left\{\sqrt{P\left(\theta_{1}, 0\right)}+\sqrt{Q\left(\theta_{1}, 0\right)}, \sqrt{P\left(\theta_{2}, 0\right)}+\sqrt{Q\left(\theta_{2}, 0\right)}, \sqrt{P(0,0)}+\sqrt{Q(0,0)}\right\}, \\
\text { if } a_{2}>a_{3} \text { and } a_{1} a_{4}-a_{2} a_{3} \neq 0, \\
\min \left\{\sqrt{P\left(\theta_{1}, 0\right)}+\sqrt{Q\left(\theta_{1}, 0\right)}, \sqrt{P\left(\theta_{3}, 0\right)}+\sqrt{Q\left(\theta_{3}, 0\right)}, \sqrt{P(0,0)}+\sqrt{Q(0,0)}\right\}, \\
\text { if } a_{2}<a_{3} \text { and } a_{1} a_{4}-a_{2} a_{3} \neq 0, \\
\min \left\{\sqrt{P\left(\theta_{4}, 0\right)}+\sqrt{Q\left(\theta_{4}, 0\right)}, \sqrt{P(0,0)}+\sqrt{Q(0,0)}\right\}, \\
\text { if } a_{2} \neq a_{3} \text { and } a_{1} a_{4}-a_{2} a_{3}=0, \\
\min \left\{\left.\sqrt{P\left(\pi-\theta_{0}, 0\right)}\right|_{a_{2}=a_{3}}, \sqrt{P\left(\theta_{1}, 0\right)}+\sqrt{Q\left(\theta_{1}, 0\right)}, \sqrt{P(0,0)}+\sqrt{Q(0,0)}\right\}, \\
\text { if } a_{2}=a_{3} .
\end{array}\right.
$$

For $\varphi=\pi, 2 \pi$, by eq. (52), there is 


$$
\begin{aligned}
\min \{\sqrt{P(\theta, \pi)}+\sqrt{Q(\theta, \pi)}\} & =\min \{\sqrt{P(\theta, 2 \pi)}+\sqrt{Q(\theta, 2 \pi)}\} \\
& =\min \{\sqrt{P(\theta, 0)}+\sqrt{Q(\theta, 0)}\} .
\end{aligned}
$$

Now we investigate the hyperplanes $\varphi=\frac{\pi}{2}$, and $\varphi=\frac{3 \pi}{2}$. From

$$
\frac{\mathrm{d}\left(\sqrt{P\left(\theta, \frac{\pi}{2}\right)}+\sqrt{Q\left(\theta, \frac{\pi}{2}\right)}\right)}{\mathrm{d} \theta}=0,
$$

there is $\cos \theta=0$, i.e. $\theta=\frac{\pi}{2}$. Similarly, by

$$
\frac{\mathrm{d}\left(\sqrt{P\left(\theta, \frac{3 \pi}{2}\right)}+\sqrt{Q\left(\theta, \frac{3 \pi}{2}\right)}\right)}{\mathrm{d} \theta}=0,
$$

we also get $\theta=\frac{\pi}{2}$. From eqs. (19) and (20), we have

$$
\begin{aligned}
\sqrt{P\left(\frac{\pi}{2}, \frac{\pi}{2}\right)}+\sqrt{Q\left(\frac{\pi}{2}, \frac{\pi}{2}\right)} & =\sqrt{P\left(\frac{\pi}{2}, \frac{3 \pi}{2}\right)}+\sqrt{Q\left(\frac{\pi}{2}, \frac{3 \pi}{2}\right)} \\
& =\sqrt{1-4 a_{2}^{2} a_{3}^{2}+8 a_{1} a_{2} a_{3} a_{4}-4 a_{0}^{2} a_{4}^{2}-4 a_{1}^{2} a_{4}^{2}} .
\end{aligned}
$$

Therefore,

$$
\begin{aligned}
& \min \{\sqrt{P(\theta, \varphi)}+\sqrt{Q(\theta, \varphi)}\} \\
= & \min \left\{\sqrt{P(0, \varphi)}+\sqrt{Q(0, \varphi)}, \sqrt{P\left(\frac{\pi}{2}, \frac{\pi}{2}\right)}+\sqrt{Q\left(\frac{\pi}{2}, \frac{\pi}{2}\right)}, \min \{\sqrt{P(\theta, 0)}+\sqrt{Q(\theta, 0)}\}\right\} .
\end{aligned}
$$

\section{$2.10 \mu=\pi$ and $a_{0} a_{1} a_{2} a_{3} a_{4} \neq 0$}

In this section, we examine the quantum channel (2) with coefficients satisfying $\mu=\pi$ and $a_{0} a_{1} a_{2} a_{3} a_{4} \neq 0$.

We begin with the special case $\sin \theta=0$. In this case, there is

$$
\begin{aligned}
\sqrt{P(0, \varphi)}+\sqrt{Q(0, \varphi)} & =\sqrt{P(\pi, \varphi)}+\sqrt{Q(\pi, \varphi)} \\
& =a_{0}^{2}+\sqrt{\left(1-a_{0}^{2}\right)^{2}-4\left(a_{1} a_{4}+a_{2} a_{3}\right)^{2}} \\
& =a_{0}^{2}+\sqrt{\left[\left(a_{2}-a_{3}\right)^{2}+\left(a_{1}-a_{4}\right)^{2}\right]\left[\left(a_{2}+a_{3}\right)^{2}+\left(a_{1}+a_{4}\right)^{2}\right]} .
\end{aligned}
$$

It is seen that $P(0, \varphi)=Q(\pi, \varphi)=a_{0}^{2} \neq 0, P(\pi, \varphi)=0$ if and only if $a_{2}=a_{3}$, and $a_{1}=a_{4}$, and $Q(0, \varphi)=0$ if and only if $a_{2}=a_{3}$ and $a_{1}=a_{4}$. Next we suppose that $\sin \theta \neq 0$, i.e. $\theta \in(0, \pi)$.

First, we look for the condition of $P(\theta, \varphi)=0, Q(\theta, \varphi)=0$. If $P(\theta, \varphi)=0$, then

$$
\begin{aligned}
\cos \varphi= & z_{1} \\
= & \frac{1}{2 a_{0} a_{1}^{2} t}\left[t^{2} a_{0}^{2} a_{1}+\left(1-a_{0}^{2}\right) a_{1}-2 a_{4}\left(a_{2} a_{3}+a_{1} a_{4}\right)\right. \\
& \left.-2 \sqrt{-t^{2} a_{0}^{2} a_{1} a_{2} a_{3} a_{4}+\left(a_{2} a_{3}+a_{1} a_{4}\right)\left(a_{1} a_{3}-a_{2} a_{4}\right)\left(a_{1} a_{2}-a_{3} a_{4}\right)}\right],
\end{aligned}
$$




$$
\begin{aligned}
\cos \varphi= & z_{2} \\
= & \frac{1}{2 a_{0} a_{1}^{2} t}\left[t^{2} a_{0}^{2} a_{1}+\left(1-a_{0}^{2}\right) a_{1}-2 a_{4}\left(a_{2} a_{3}+a_{1} a_{4}\right)\right. \\
& \left.+2 \sqrt{-t^{2} a_{0}^{2} a_{1} a_{2} a_{3} a_{4}+\left(a_{2} a_{3}+a_{1} a_{4}\right)\left(a_{1} a_{3}-a_{2} a_{4}\right)\left(a_{1} a_{2}-a_{3} a_{4}\right)}\right] .
\end{aligned}
$$

Here $\left(a_{1} a_{3}-a_{2} a_{4}\right)\left(a_{1} a_{2}-a_{3} a_{4}\right)>0$, and

$$
t \in\left(0, \sqrt{\frac{\left(a_{2} a_{3}+a_{1} a_{4}\right)\left(a_{1} a_{3}-a_{2} a_{4}\right)\left(a_{1} a_{2}-a_{3} a_{4}\right)}{a_{0}^{2} a_{1} a_{2} a_{3} a_{4}}}\right],
$$

because $\left(a_{1} a_{3}-a_{2} a_{4}\right)\left(a_{1} a_{2}-a_{3} a_{4}\right) \leqslant 0$ implies that $P(\theta, \varphi) \neq 0$. Directly,

$$
\left(a_{1} a_{3}-a_{2} a_{4}\right)\left(a_{1} a_{2}-a_{3} a_{4}\right)>0
$$

if and only if $a_{2}^{2}+a_{3}^{2}<\frac{a_{2} a_{3}\left(a_{1}^{2}+a_{4}^{2}\right)}{a_{1} a_{4}}$.

Note that

$$
\left(1-2 a_{0}^{2}\right) a_{1}-2 a_{4}\left(a_{2} a_{3}+a_{1} a_{4}\right)=a_{1}\left(a_{1}^{2}-a_{4}^{2}\right)+a_{1}\left(a_{2}^{2}+a_{3}^{2}\right)-2 a_{2} a_{3} a_{4}>0
$$

in the case of $a_{1}>a_{4}$,

$$
\begin{aligned}
\left(1-2 a_{0}^{2}\right) a_{1}-2 a_{4}\left(a_{2} a_{3}+a_{1} a_{4}\right) & =a_{1}\left(a_{1}^{2}-a_{4}^{2}\right)+a_{1}\left(a_{2}^{2}+a_{3}^{2}\right)-2 a_{2} a_{3} a_{4} \\
& <\frac{\left(a_{1}^{2}-a_{4}^{2}\right)\left(a_{2} a_{3}+a_{1} a_{4}\right)}{a_{4}} \\
& <0
\end{aligned}
$$

in the case of $a_{1}<a_{4}$ and $\left(a_{1} a_{3}-a_{2} a_{4}\right)\left(a_{1} a_{2}-a_{3} a_{4}\right)>0$, and

$$
\begin{aligned}
& {\left[\left(1-a_{0}^{2}\right) a_{1}-2 a_{4}\left(a_{2} a_{3}+a_{1} a_{4}\right)\right]^{2}-\left\{2 \sqrt{\left(a_{2} a_{3}+a_{1} a_{4}\right)\left(a_{1} a_{3}-a_{2} a_{4}\right)\left(a_{1} a_{2}-a_{3} a_{4}\right)}\right\}^{2} } \\
= & a_{1}^{2}\left[\left(a_{1}-a_{4}\right)^{2}+\left(a_{2}-a_{3}\right)^{2}\right]\left[\left(a_{1}+a_{4}\right)^{2}+\left(a_{2}+a_{3}\right)^{2}\right] \\
> & 0 .
\end{aligned}
$$

It follows that

$$
\left(1-a_{0}^{2}\right) a_{1}-2 a_{4}\left(a_{2} a_{3}+a_{1} a_{4}\right)+2 \sqrt{\left(a_{2} a_{3}+a_{1} a_{4}\right)\left(a_{1} a_{3}-a_{2} a_{4}\right)\left(a_{1} a_{2}-a_{3} a_{4}\right)}>0
$$

and

$$
\left(1-a_{0}^{2}\right) a_{1}-2 a_{4}\left(a_{2} a_{3}+a_{1} a_{4}\right)-2 \sqrt{\left(a_{2} a_{3}+a_{1} a_{4}\right)\left(a_{1} a_{3}-a_{2} a_{4}\right)\left(a_{1} a_{2}-a_{3} a_{4}\right)}>0
$$

in the case of $a_{1}>a_{4}$;

$$
\left(1-a_{0}^{2}\right) a_{1}-2 a_{4}\left(a_{2} a_{3}+a_{1} a_{4}\right)+2 \sqrt{\left(a_{2} a_{3}+a_{1} a_{4}\right)\left(a_{1} a_{3}-a_{2} a_{4}\right)\left(a_{1} a_{2}-a_{3} a_{4}\right)}<0
$$

and

$$
\left(1-a_{0}^{2}\right) a_{1}-2 a_{4}\left(a_{2} a_{3}+a_{1} a_{4}\right)-2 \sqrt{\left(a_{2} a_{3}+a_{1} a_{4}\right)\left(a_{1} a_{3}-a_{2} a_{4}\right)\left(a_{1} a_{2}-a_{3} a_{4}\right)}<0
$$

in the case of $a_{1}<a_{4}$. Thus, if $a_{1}>a_{4}$, then both $z_{1}$ and $z_{2}$ go to $+\infty$ when $t \rightarrow 0$; if $a_{1}<a_{4}$, then both $z_{1}$ and $z_{2}$ go to $-\infty$ when $t \rightarrow 0$.

Now suppose that $a_{1}>a_{4}$. From $z_{1}=1$, there is

$$
\left[t^{2} a_{0}^{2} a_{1}-2 t a_{0} a_{1}^{2}+\left(1-a_{0}^{2}\right) a_{1}-2 a_{4}\left(a_{2} a_{3}+a_{1} a_{4}\right)\right]^{2}
$$




$$
-\left[2 \sqrt{-t^{2} a_{0}^{2} a_{1} a_{2} a_{3} a_{4}+\left(a_{2} a_{3}+a_{1} a_{4}\right)\left(a_{1} a_{3}-a_{2} a_{4}\right)\left(a_{1} a_{2}-a_{3} a_{4}\right)}\right]^{2}=0,
$$

and the solutions of eq. (93) are

$$
\begin{aligned}
& t=t_{11}=\frac{a_{1}+a_{4}-\sqrt{-\left(a_{2}+a_{3}\right)^{2}}}{a_{0}}, \quad t=t_{12}=\frac{a_{1}+a_{4}+\sqrt{-\left(a_{2}+a_{3}\right)^{2}}}{a_{0}}, \\
& t=t_{13}=\frac{a_{1}-a_{4}-\sqrt{-\left(a_{2}-a_{3}\right)^{2}}}{a_{0}}, \quad t=t_{14}=\frac{a_{1}-a_{4}+\sqrt{-\left(a_{2}-a_{3}\right)^{2}}}{a_{0}} .
\end{aligned}
$$

Here, $t_{11}$ and $t_{12}$ are imaginary numbers, and $t_{13}$ and $t_{14}$ are positive real numbers only if $a_{2}=a_{3}$, and $a_{1}>a_{4}$. It is not difficult to check that $\theta_{0}$ is a root of $z_{1}=1$ and the minimum point of $z_{1}$ in the case of $a_{1}>a_{4}$ and $a_{2}=a_{3}$. Here $t=\cot \frac{\theta_{0}}{2}=\frac{a_{1}-a_{4}}{a_{0}} . z_{1} \geqslant 1$ comes directly because $z=z_{1}$ is a continuous function of $t$, goes to $+\infty$ when $t \rightarrow 0$, and has only one intersection point with the straight line $z=1$, where the equality $z_{1}=1$ holds iff $a_{2}=a_{3}$, $a_{1}>a_{4}$ and $\cot \frac{\theta_{0}}{2}=\frac{a_{1}-a_{4}}{a_{0}}$. We show that $z_{2}>1$ in the case of $a_{1}>a_{4}$ in the same way.

Similarly, we prove that if $a_{1}<a_{4}$, then $z_{1}<-1$, and $z_{2} \leqslant-1$, where $z_{2}=-1$ iff $a_{2}=a_{3}$, $a_{1}<a_{4}$, and $t=\cot \frac{\bar{\theta}_{0}}{2}=\frac{-a_{1}+a_{4}}{a_{0}}$. Therefore, $P(\theta, \varphi)=0$ iff $\varphi=0$ and $\theta=\theta_{0}$ in the case of $a_{2}=a_{3}$, and $a_{1}>a_{4}$, or $\varphi=\pi$ and $\theta=\bar{\theta}_{0}$ in the case of $a_{2}=a_{3}$ and $a_{1}<a_{4}$. It is derived that

$$
\begin{aligned}
\left.(\sqrt{P(\theta, \varphi)}+\sqrt{Q(\theta, \varphi)})\right|_{P(\theta, \varphi)=0, \theta \in(0, \pi)} & =\left.\sqrt{Q\left(\theta_{0}, 0\right)}\right|_{\left\{a_{2}=a_{3}, a_{1}>a_{4}\right\}} \\
& =\left.\sqrt{Q\left(\bar{\theta}_{0}, \pi\right)}\right|_{\left\{a_{2}=a_{3}, a_{1}<a_{4}\right\}} \\
& =\sqrt{\left(1-2 a_{4}^{2}\right)^{2}+4 a_{3}^{2}\left(-1+a_{1}^{2}+a_{3}^{2}-2 a_{1} a_{4}+3 a_{4}^{2}\right)} .
\end{aligned}
$$

Immediately, from eqs. (19) and (20), $Q(\theta, \varphi)=0$ iff $\varphi=\pi$ and $\theta=\pi-\theta_{0}$ in the case of $a_{2}=a_{3}$ and $a_{1}>a_{4}$, or $\varphi=0$ and $\theta=\pi-\bar{\theta}_{0}$ in the case of $a_{2}=a_{3}$ and $a_{1}<a_{4}$, and

$$
\left.(\sqrt{P(\theta, \varphi)}+\sqrt{Q(\theta, \varphi)})\right|_{Q(\theta, \varphi)=0}=\left.(\sqrt{P(\theta, \varphi)}+\sqrt{Q(\theta, \varphi)})\right|_{Q(\theta, \varphi)=0} .
$$

From above, it is seen that the quantum channel (2), the coefficients of which satisfy $\mu=\pi$, $a_{0} a_{1} a_{2} a_{3} a_{4} \neq 0$ and $a_{2}=a_{3}$, is collapsed to a Bell state with probability $p_{1}=p_{2}=$ $\frac{a_{0}^{2}\left(1-a_{0}^{2}+3 a_{1}^{2}-4 a_{1} a_{4}+a_{4}^{2}\right)}{a_{0}^{2}+\left(a_{1}-a_{4}\right)^{2}}$ by Charlie's measurement in the basis (3) with $(\theta, \varphi)=\left(\theta_{0}, 0\right)$ or $(\theta, \varphi)=\left(\pi-\theta_{0}, \pi\right)$ in the case of $a_{1}>a_{4}$, or $(\theta, \varphi)=\left(\bar{\theta}_{0}, \pi\right)$, or $(\theta, \varphi)=\left(\pi-\bar{\theta}_{0}, 0\right)$ in the case of $a_{1}<a_{4}$. In particular, this quantum channel is also purified to an EPR pair with probability $2 a_{1}^{2}+2 a_{2}^{2}$ via the controller's measurement in the basis $\{|0\rangle,|1\rangle\}$ in the case of $a_{1}=a_{4}$. 
In the following, we suppose that $P(\theta, \varphi) Q(\theta, \varphi) \neq 0$. In order to obtain the minimum of $\sqrt{P(\theta, \varphi)}+\sqrt{Q(\theta, \varphi)}$, we need to find the points such that eqs. (26) and (27) hold. From eq. (26), we get $\sin \varphi=0$, or

$$
\begin{gathered}
y a_{0}\left[2 a_{1} a_{2}^{2} a_{3}^{2}-a_{2} a_{3} a_{4}\left(-2 a_{0}^{2}-4 a_{1}^{2}-2 a_{4}^{2}+1\right)-2 a_{1} a_{4}^{2}\left(a_{2}^{2}+a_{3}^{2}\right)\right] \\
-2 \cos \varphi\left(a_{2} a_{3}+a_{1} a_{4}\right)\left(a_{1} a_{3}-a_{2} a_{4}\right)\left(a_{1} a_{2}-a_{3} a_{4}\right)=0 .
\end{gathered}
$$

By eq. (27), we have $\sin \varphi=0$, or

$$
\begin{aligned}
& 4 y^{3} a_{0}^{3}\left(a_{2} a_{3}+a_{1} a_{4}\right)\left(a_{1} a_{3}-a_{2} a_{4}\right)\left(a_{1} a_{2}-a_{3} a_{4}\right) \\
& -8 y^{2} a_{0}^{2} a_{1} \cos \varphi\left(a_{2} a_{3}+a_{1} a_{4}\right)\left(a_{1} a_{3}-a_{2} a_{4}\right)\left(a_{1} a_{2}-a_{3} a_{4}\right) \\
& +y a_{0}\left[\left(a_{1}-2 a_{2} a_{3} a_{4}-2 a_{1} a_{4}^{2}\right)\right. \\
& \times\left(2 a_{1} a_{2}^{2} a_{3}^{2}-a_{2} a_{3} a_{4}+4 a_{1}^{2} a_{2} a_{3} a_{4}-2 a_{1} a_{2}^{2} a_{4}^{2}-2 a_{1} a_{3}^{2} a_{4}^{2}+2 a_{2} a_{3} a_{4}^{3}\right) \\
& \left.+4 a_{1}^{2} \cos ^{2} \varphi\left(a_{2} a_{3}+a_{1} a_{4}\right)\left(a_{1} a_{3}-a_{2} a_{4}\right)\left(a_{1} a_{2}-a_{3} a_{4}\right)\right] \\
& +2 \cos \varphi\left(-a_{1}+2 a_{2} a_{3} a_{4}+2 a_{1} a_{4}^{2}\right) \\
& \times\left[a_{2}^{2} a_{3}^{2}\left(a_{1}^{2}+a_{4}^{2}\right)-a_{1} a_{2} a_{3} a_{4}\left(-2 a_{1}^{2}-2 a_{4}^{2}+1\right)-a_{1}^{2}\left(a_{2}^{2}+a_{3}^{2}\right) a_{4}^{2}\right] \\
& =0 .
\end{aligned}
$$

If $\left(a_{1} a_{3}-a_{2} a_{4}\right)\left(a_{1} a_{2}-a_{3} a_{4}\right)=0$, then eqs. (97) and (98) become

$$
y=0
$$

and

$$
y\left[a_{4}^{2}-\left(1-2 a_{0}^{2}\right) a_{1}^{2}\right]-2 a_{0} a_{1} \cos \varphi\left(a_{1}^{2}+a_{4}^{2}\right)=0 .
$$

However, if $\left(a_{1} a_{3}-a_{2} a_{4}\right)\left(a_{1} a_{2}-a_{3} a_{4}\right) \neq 0$, then eqs. (97) and (98) become

$$
\cos \varphi=\frac{y a_{0}\left\{-2 a_{1} a_{4}^{2}\left(a_{2}^{2}+a_{3}^{2}\right)+a_{2} a_{3}\left[2 a_{1} a_{2} a_{3}+a_{4}+2\left(a_{1}^{2}-a_{2}^{2}-a_{3}^{2}\right) a_{4}\right]\right\}}{2\left(a_{2} a_{3}+a_{1} a_{4}\right)\left(a_{1} a_{3}-a_{2} a_{4}\right)\left(a_{1} a_{2}-a_{3} a_{4}\right)}
$$

and

$$
\frac{y\left(1+y^{2}\right) a_{0}^{3} a_{2}^{2} a_{3}^{2} a_{4}^{2}\left[2 a_{2} a_{3} a_{4}-a_{1}\left(1-2 a_{4}^{2}\right)\right]^{2}}{\left(a_{2} a_{3}+a_{1} a_{4}\right)\left(a_{1} a_{3}-a_{2} a_{4}\right)\left(a_{1} a_{2}-a_{3} a_{4}\right)}=0 .
$$

That is, $y=0$, and $\cos \varphi=0$.

For getting the minimum point of $\sqrt{P(\theta, \varphi)}+\sqrt{Q(\theta, \varphi)}$, it is enough for us to consider the hyperplanes $\sin \varphi=0$, and $\cos \varphi=0$. For hyperplane $\sin \varphi=0$, we only need to consider the case $\varphi=0$ by eqs. (19)-(21). For hyperplane $\cos \varphi=0$, we only need to consider the case $\varphi=\frac{\pi}{2}$ by eqs. (19) and (20).

Now, we consider the case $\varphi=0$. In this case eq. (28) becomes

$$
\begin{aligned}
& {\left[-2 a_{1} a_{2} a_{3}+a_{4}-2 a_{1}^{2} a_{4}+2 y a_{0}\left(a_{2} a_{3}+a_{1} a_{4}\right)\right]} \\
& \times\left[4 y^{2} a_{0}^{2}\left(a_{1} a_{3}-a_{2} a_{4}\right)\left(a_{1} a_{2}-a_{3} a_{4}\right)+2 y a_{0}\left(2 a_{1} a_{2} a_{3}-4 a_{1}^{3} a_{2} a_{3}-2 a_{1} a_{2}^{3} a_{3}\right.\right. \\
& -2 a_{1} a_{2} a_{3}^{3}-a_{2}^{2} a_{4}+4 a_{1}^{2} a_{2}^{2} a_{4}+2 a_{2}^{4} a_{4}-a_{3}^{2} a_{4}
\end{aligned}
$$




$$
\begin{aligned}
& \left.+4 a_{1}^{2} a_{3}^{2} a_{4}+2 a_{3}^{4} a_{4}-8 a_{1} a_{2} a_{3} a_{4}^{2}+2 a_{2}^{2} a_{4}^{3}+2 a_{3}^{2} a_{4}^{3}\right) \\
& \left.+\left(a_{2}-2 a_{1}^{2} a_{2}-2 a_{2}^{3}+2 a_{1} a_{3} a_{4}-2 a_{2} a_{4}^{2}\right)\left(a_{3}-2 a_{1}^{2} a_{3}-2 a_{3}^{3}+2 a_{1} a_{2} a_{4}-2 a_{3} a_{4}^{2}\right)\right] \\
& =0 .
\end{aligned}
$$

If $\left(a_{1} a_{3}-a_{2} a_{4}\right)\left(a_{1} a_{2}-a_{3} a_{4}\right) \neq 0$, then

$$
\begin{aligned}
& y=\cot \theta_{1}=\frac{2 a_{1} a_{2} a_{3}-a_{4}+2 a_{1}^{2} a_{4}}{2 a_{0}\left(a_{2} a_{3}+a_{1} a_{4}\right)} \\
& y=\cot \theta_{2}=\frac{-a_{2}+2 a_{1}^{2} a_{2}+2 a_{2}^{3}-2 a_{1} a_{3} a_{4}+2 a_{2} a_{4}^{2}}{2 a_{0}\left(a_{1} a_{2}-a_{3} a_{4}\right)}, \\
& y=\cot \theta_{3}=\frac{-a_{3}+2 a_{1}^{2} a_{3}+2 a_{3}^{3}-2 a_{1} a_{2} a_{4}+2 a_{3} a_{4}^{2}}{2 a_{0}\left(a_{1} a_{3}-a_{2} a_{4}\right)},
\end{aligned}
$$

where $\theta_{1}$ is a root of eq. (24), while $\theta_{2}$ is a root of eq. (24) only in the case of $a_{2}<a_{3}$, and $\theta_{3}$ is a root of eq. (24) only in the case of $a_{2}>a_{3}$. If $\left(a_{1} a_{2}-a_{3} a_{4}\right)=0$, and $a_{1} \neq a_{4}$, then $a_{2} \neq a_{3}$, and

$$
y=\cot \theta_{4}=\frac{-a_{1}+2 a_{1}^{3}+2 a_{1} a_{3}^{2}}{2 a_{0}\left(a_{1}^{2}+a_{3}^{2}\right)}, \quad y=\cot \theta_{5}=\frac{-a_{1}+2 a_{1}^{3}+2 a_{1} a_{3}^{2}}{2 a_{0}\left(a_{1}^{2}-a_{4}^{2}\right)},
$$

where $\theta_{4}$ is a root of eq. (24), while $\theta_{5}$ is a root of eq. (24) only if $a_{2}>a_{3}$. If $\left(a_{1} a_{3}-a_{2} a_{4}\right)=0$, and $a_{1} \neq a_{4}$, then $a_{2} \neq a_{3}$, and

$$
y=\cot \theta_{6}=\frac{-a_{1}+2 a_{1}^{3}+2 a_{1} a_{2}^{2}}{2 a_{0}\left(a_{1}^{2}+a_{2}^{2}\right)}, \quad y=\cot \theta_{7}=\frac{-a_{1}+2 a_{1}^{3}+2 a_{1} a_{2}^{2}}{2 a_{0}\left(a_{1}^{2}-a_{4}^{2}\right)},
$$

where $\theta_{6}$ is a root of eq. (24), while $\theta_{7}$ is a root only if $a_{2}<a_{3}$. If $a_{1}=a_{4}$, and $a_{2}=a_{3}$, then

$$
y=\cot \theta_{8}=-\frac{a_{0} a_{1}}{2\left(a_{1}^{2}+a_{2}^{2}\right)},
$$

where $\theta_{8}$ is a root of eq. (24).

Therefore,

$$
\min \{\sqrt{P(\theta, 0)}+\sqrt{Q(\theta, 0)}\}=\left\{\begin{array}{lll}
\min \left\{\left.(\sqrt{P(\theta, 0)}+\sqrt{Q(\theta, 0)})\right|_{a_{2}>a_{3}}\right\}, & \text { if } & a_{2}>a_{3}, \\
\min \left\{\left.(\sqrt{P(\theta, 0)}+\sqrt{Q(\theta, 0)})\right|_{a_{2}<a_{3}}\right\}, & \text { if } & a_{2}<a_{3}, \\
\min \left\{\left.(\sqrt{P(\theta, 0)}+\sqrt{Q(\theta, 0)})\right|_{a_{2}=a_{3}}\right\}, & \text { if } & a_{2}=a_{3} .
\end{array}\right.
$$

Here,

$$
=\left\{\begin{array}{c}
\min \left\{\left.(\sqrt{P(\theta, 0)}+\sqrt{Q(\theta, 0)})\right|_{a_{2}>a_{3}}\right\} \\
\min \left\{\sqrt{P\left(\theta_{1}, 0\right)}+\sqrt{Q\left(\theta_{1}, 0\right)}, \sqrt{P\left(\theta_{3}, 0\right)}+\sqrt{Q\left(\theta_{3}, 0\right)}, \sqrt{P(0,0)}+\sqrt{Q(0,0)}\right\}, \\
\text { if }\left(a_{1} a_{3}-a_{2} a_{4}\right)\left(a_{1} a_{2}-a_{3} a_{4}\right) \neq 0, \\
\min \left\{\sqrt{P\left(\theta_{4}, 0\right)}+\sqrt{Q\left(\theta_{4}, 0\right)}, \sqrt{P\left(\theta_{5}, 0\right)}+\sqrt{Q\left(\theta_{5}, 0\right)}, \sqrt{P(0,0)}+\sqrt{Q(0,0)}\right\}, \\
\text { if } a_{1} a_{2}-a_{3} a_{4}=0, \quad \text { and } \quad a_{1} \neq a_{4}, \\
\min \left\{\sqrt{P\left(\theta_{6}, 0\right)}+\sqrt{Q\left(\theta_{6}, 0\right)}, \sqrt{P(0,0)}+\sqrt{Q(0,0)}\right\}, \\
\text { if } a_{1} a_{3}-a_{2} a_{4}=0, \quad \text { and } a_{1} \neq a_{4} ;
\end{array}\right.
$$




$$
\begin{aligned}
& \min \left\{\left.(\sqrt{P(\theta, 0)}+\sqrt{Q(\theta, 0)})\right|_{a_{2}<a_{3}}\right\} \\
& \min \left\{\sqrt{P\left(\theta_{1}, 0\right)}+\sqrt{Q\left(\theta_{1}, 0\right)}, \sqrt{P\left(\theta_{2}, 0\right)}+\sqrt{Q\left(\theta_{2}, 0\right)}, \sqrt{P(0,0)}+\sqrt{Q(0,0)}\right\}, \\
& \text { if }\left(a_{1} a_{3}-a_{2} a_{4}\right)\left(a_{1} a_{2}-a_{3} a_{4}\right) \neq 0 \text {, } \\
& =\left\{\min \left\{\sqrt{P\left(\theta_{4}, 0\right)}+\sqrt{Q\left(\theta_{4}, 0\right)}, \sqrt{P(0,0)}+\sqrt{Q(0,0)}\right\},\right. \\
& \text { if } a_{1} a_{2}-a_{3} a_{4}=0 \text {, and } a_{1} \neq a_{4} \text {, } \\
& \min \left\{\sqrt{P\left(\theta_{6}, 0\right)}+\sqrt{Q\left(\theta_{6}, 0\right)}, \sqrt{P\left(\theta_{7}, 0\right)}+\sqrt{Q\left(\theta_{7}, 0\right)}, \sqrt{P(0,0)}+\sqrt{Q(0,0)}\right\}, \\
& \text { if } a_{1} a_{3}-a_{2} a_{4}=0 \text {, and } a_{1} \neq a_{4} \text {; } \\
& \min \left\{\left.(\sqrt{P(\theta, 0)}+\sqrt{Q(\theta, 0)})\right|_{a_{2}=a_{3}}\right\} \\
& = \begin{cases}\min \left\{\sqrt{P\left(\theta_{1}, 0\right)}+\sqrt{Q\left(\theta_{1}, 0\right)},\left.\sqrt{Q\left(\theta_{0}, 0\right)}\right|_{a_{2}=a_{3}, a_{1}>a_{4}}, \sqrt{P(0,0)}+\sqrt{Q(0,0)}\right\}, & \text { if } a_{1}>a_{4}, \\
\min \left\{\sqrt{P\left(\theta_{1}, 0\right)}+\sqrt{Q\left(\theta_{1}, 0\right)},\left.\sqrt{Q\left(\bar{\theta}_{0}, \pi\right)}\right|_{a_{2}=a_{3}, a_{1}<a_{4}}, \sqrt{P(0,0)}+\sqrt{Q(0,0)}\right\}, & \text { if } \quad a_{1}<a_{4}, \\
\min \left\{\sqrt{P\left(\theta_{8}, 0\right)}+\sqrt{Q\left(\theta_{8}, 0\right)}, a_{0}^{2}\right\}, & \text { if } \quad a_{1}=a_{4} .\end{cases}
\end{aligned}
$$

By eqs. (19) - (21), we have

$$
\min \{\sqrt{P(\theta, \pi)}+\sqrt{Q(\theta, \pi)}\}=\min \{\sqrt{P(\theta, 0)}+\sqrt{Q(\theta, 0)}\}=\min \{\sqrt{P(\theta, 2 \pi)}+\sqrt{Q(\theta, 2 \pi)}\} .
$$

Now we examine the case $\varphi=\frac{\pi}{2}$. In this case, eq. (28) goes to

$$
4 y a_{0}^{4}\left[1-2 a_{0}^{2}-4\left(a_{2} a_{3}+a_{1} a_{4}\right)^{2}\right]\left[a_{2}^{2} a_{3}^{2}+2 a_{1} a_{2} a_{3} a_{4}-a_{4}^{2}\left(a_{2}^{2}+a_{3}^{2}\right)\right]\left(1+y^{2}\right)^{-3 / 2}=0,
$$

which implies that $\theta=\frac{\pi}{2}$. It is shown that $\theta=\frac{\pi}{2}$ is the only possible extreme point of both

$$
\begin{aligned}
& \sqrt{P\left(\theta, \frac{\pi}{2}\right)}+\sqrt{Q\left(\theta, \frac{\pi}{2}\right)} \text { and } \sqrt{P\left(\theta, \frac{3 \pi}{2}\right)}+\sqrt{Q\left(\theta, \frac{3 \pi}{2}\right)} \text {, and } \\
& \quad \sqrt{P\left(\frac{\pi}{2}, \frac{\pi}{2}\right)}+\sqrt{Q\left(\frac{\pi}{2}, \frac{\pi}{2}\right)}=\sqrt{P\left(\frac{\pi}{2}, \frac{3 \pi}{2}\right)}+\sqrt{Q\left(\frac{\pi}{2}, \frac{3 \pi}{2}\right)}=\sqrt{1-4\left(a_{2} a_{3}+a_{1} a_{4}\right)^{2}-4 a_{0}^{2} a_{4}^{2}} .
\end{aligned}
$$

To sum up,

$$
\begin{aligned}
& \min \{\sqrt{P(\theta, \varphi)}+\sqrt{Q(\theta, \varphi)}\} \\
= & \min \left\{\sqrt{P(0, \varphi)}+\sqrt{Q(0, \varphi)}, \sqrt{P\left(\frac{\pi}{2}, \frac{\pi}{2}\right)}+\sqrt{Q\left(\frac{\pi}{2}, \frac{\pi}{2}\right)}, \min \{\sqrt{P(\theta, 0)}+\sqrt{Q(\theta, 0)}\},\right. \\
& \left.\left.(\sqrt{P(\theta, \varphi)}+\sqrt{Q(\theta, \varphi)})\right|_{P(\theta, \varphi)=0}\right\} .
\end{aligned}
$$

\section{Conclusion}

In brief, we give the analytic expression of the localizable entanglement (LE), i.e. the maximal probability of successful controlled teleportation of an unknown qubit state (14) via every three-qubit state (2) satisfying $a_{1} a_{2} a_{3} a_{4} \sin \mu=0$, and investigate how to achieve it (that is, Charlie finds the optimal measurement basis, performs optimal measurements, and communicates the results). 
The authors thank Prof. J. I. Cirac for his fruitful discussions and hospitality during our stay at Max-Planck-Institut für Quantenoptik.

1 Bennett C H, Brassard G, Crépeau C, et al. Teleporting an unknown quantum state via dual classical and Einstein-PodolskyRosen channels. Phys Rev Lett, 1993, 70(13): 1895-1899

2 Fujii M. Continuous-variable quantum teleportation with a conventional laser. Phys Rev A, 2003, 68(5): 050302

3 An N B. Teleportation of coherent-state superpositions within a network. Phys Rev A, 2003, 68(2): 022321

4 Bowen W P, Treps N, Buchler B C, et al. Experimental investigation of continuous-variable quantum teleportation. Phys Rev A, 2003, 67(3): 032302

5 Johnson T J, Bartlett S D, Sanders B C. Continuous-variable quantum teleportation of entanglement. Phys Rev A, 2002, 66(4): 042326

6 Vaidman L. Teleportation of quantum states. Phys Rev A, 1994, 49(2): 1473-1476

7 Braunstein S L, Kimble H J. Teleportation of continuous quantum variables. Phys Rev Lett, 1998, 80(4): 869-872

8 Son W, Lee J, Kim M S, et al. Conclusive teleportation of a d-dimensional unknown state. Phys Rev A, 2001, 64(6): 064304

9 Bruß D, DiVincenzo D P, Ekert A, et al. Optimal universal and state-dependent quantum cloning. Phys Rev A, 1998, 57(4): $2368-2378$

10 Gordon G, Rigolin G. Generalized teleportation protocol. Phys Rev A, 2006, 73(4): 042309

11 Gao T, Yan F L, Wang Z X. Quantum networks for probabilistic teleportation of many particle state of general form. Quant Inform Comp, 2004, 4(3): 186-195

12 Karlsson A, Bourennane M. Quantum teleportation using three-particle entanglement. Phys Rev A, 1998, 58(6): 4394-4400

13 Yang C P, Chu S I, Han S. Efficient many-party controlled teleportation of multiqubit quantum information via entanglement. Phys Rev A, 2004, 70(2): 022329

14 Pati A K. Assisted cloning and orthogonal complementing of an unknown state. Phys Rev A, 2000, 61(2): 022308

15 Agrawal P, Pati A K. Probabilistic quantum teleportation. Phys Lett A, 2002, 305(1-2): 12-17

16 Pati A K, Agrawal P. Probabilistic teleportation and quantum operation. J Opt B: Quant Semiclass Opt, 2004, 6(8): 844-848

17 Yan F L, Wang D. Probabilistic and controlled teleportation of unknown quantum states. Phys Lett A, 2003, 316(5): 297-303

18 Deng F G, Li C Y, Li Y S, et al. Symmetric multiparty-controlled teleportation of an arbitrary two-particle entanglement. Phys Rev A, 2005, 72(2): 022338

19 Long G L, Deng F G, Wang C, et al. Quantum secure direct communication and deterministic secure quantum communication. Front Phys China, 2007, 2(3): 251-272

20 Wang X B, Hiroshima T, Tomita A, et al. Quantum information with Gaussian states. Phys Rep, 2007, 448(1-4): 1-111

21 Yan F L, Zhang X Q. A scheme for secure direct communication using EPR pairs and teleportation. Eur Phys J B, 2004, 41(1): $75-78$

22 Bouwmeester D, Pan J W, Mattle K, et al. Experimental quantum teleportation. Nature, 1997, 390(6660): 575 - 579

23 Furusawa A, Sørensen J L, Braunstein S L, et al. Unconditional quantum teleportation. Science, 1998, 282(5389): $706-709$

24 Nielsen M A, Knill E, Laflamme R. Complete quantum teleportation using nuclear magnetic resonance. Nature, 1998, 396(6706): 52-55

25 Hillery M, Bužek V, Berthiaume A. Quantum secret sharing. Phys Rev A, 1999, 59(3): 1829-1834

26 Yan F L, Gao T, Li Y C. Quantum secret sharing between multiparty and multiparty with four states. Sci China Ser G-Phys Mech Astron, 2007, 50(5): 572-580

27 Aoun B, Tarifi M. Quantum networking. arXiv: quant-ph/0401076

28 Biham E, Huttner B, Mor T. Quantum cryptographic network based on quantum memories. Phys Rev A, 1996, 54(4): 26512658

29 Townsend P D. Quantum cryptography on multiuser optical fibre networks. Nature, 1997, 385(6611): 47-49

30 Bose S, Vedral V, Knight P L. Multiparticle generalization of entanglement swapping. Phys Rev A, 1998, 57(2): 822 - 829

31 Gao T. Controlled and secure direct communication using GHZ state and teleportation. Z Naturforsch, 2004, 59a(9): 597601

32 Ding S C, Jin Z. Review on the study of entanglement in quantum computation speedup. Chin Sci Bull, 2007, 52(16): 21612166

33 Verstraete F, Popp M, Cirac J I. Entanglement versus correlations in spin systems. Phys Rev Lett, 2004, 92(2): 027901

34 Gao T, Yan F L, Li Y C. Optimal controlled teleportation. arXiv: quant-ph/0710.1055

35 Acín A, Andrianov A, Costa L, et al. Generalized Schmidt decomposition and classification of three-quantum-bit states. Phys Rev Lett, 2000, 85(7): 1560-1563 